\title{
mTOR-mediated dedifferentiation of the retinal pigment epithelium initiates photoreceptor degeneration in mice
}

\author{
Chen Zhao, ${ }^{1}$ Douglas Yasumura, ${ }^{2}$ Xiyan Li, ${ }^{1}$ Michael Matthes, ${ }^{2}$ Marcia Lloyd, ${ }^{3}$ \\ Gregory Nielsen, ${ }^{2}$ Kelly Ahern, ${ }^{2}$ Michael Snyder, ${ }^{1}$ Dean Bok, ${ }^{3}$ Joshua L. Dunaief, ${ }^{4}$ \\ Matthew M. LaVail, ${ }^{2}$ and Douglas Vollrath ${ }^{1}$
}

\begin{abstract}
1Department of Genetics, Stanford University School of Medicine, Stanford, California, USA. ${ }^{2}$ Beckman Vision Center, University of California San Francisco, San Francisco, California, USA. 3Jules Stein Eye Institute, David Geffen School of Medicine, University of California Los Angeles, Los Angeles, California, USA. 4F.M. Kirby Center for Molecular Ophthalmology, Scheie Eye Institute, University of Pennsylvania, Philadelphia, Pennsylvania, USA.
\end{abstract}

\begin{abstract}
Retinal pigment epithelial (RPE) cell dysfunction plays a central role in various retinal degenerative diseases, but knowledge is limited regarding the pathways responsible for adult RPE stress responses in vivo. RPE mitochondrial dysfunction has been implicated in the pathogenesis of several forms of retinal degeneration. Here we have shown that postnatal ablation of RPE mitochondrial oxidative phosphorylation in mice triggers gradual epithelium dedifferentiation, typified by reduction of RPE-characteristic proteins and cellular hypertrophy. The electrical response of the retina to light decreased and photoreceptors eventually degenerated. Abnormal RPE cell behavior was associated with increased glycolysis and activation of, and dependence upon, the hepatocyte growth factor/met proto-oncogene pathway. RPE dedifferentiation and hypertrophy arose through stimulation of the AKT/mammalian target of rapamycin (AKT/mTOR) pathway. Administration of an oxidant to wild-type mice also caused RPE dedifferentiation and mTOR activation. Importantly, treatment with the $\mathrm{mTOR}$ inhibitor rapamycin blunted key aspects of dedifferentiation and preserved photoreceptor function for both insults. These results reveal an in vivo response of the mature RPE to diverse stressors that prolongs RPE cell survival at the expense of epithelial attributes and photoreceptor function. Our findings provide a rationale for $\mathrm{mTOR}$ pathway inhibition as a therapeutic strategy for retinal degenerative diseases involving RPE stress.
\end{abstract}

\section{Introduction}

The retinal pigment epithelium (RPE) is a polarized, cuboidal epithelial cell layer situated in the outer retina between the photoreceptors and choroidal vasculature. The RPE supplies an estimated $60 \%$ of the glucose consumed by the neural retina (1) and performs a variety of other functions crucial for retinal homeostasis, including delivery of amino acids and docosahexaenoic acid for photoreceptor protein and membrane synthesis; transport, storage, and enzymatic conversion of retinoids essential for phototransduction; regulation of fluid and ion balance in the subretinal space; maintenance of the blood retinal barrier; secretion of growth factors; and phagocytosis of shed photoreceptor outer segment membranes (2). The RPE is a postmitotic tissue, so RPE cells must carry out these functions for the life of an individual.

The retinal degenerative consequences of mutations in RPEexpressed genes illustrate the importance of the RPE for photoreceptor viability in humans. Mutations that impair production of the chromophore 11-cis retinal cause Leber congenital amaurosis, retinitis pigmentosa (RP), and allied disorders (3). Disruption of RPE phagocytosis causes RP and rod/cone dystrophy $(4,5)$. Mutations that affect ion channel function cause disease of the specialized retinal region necessary for high-acuity vision (the macula) (6) as well as RP (7), while mutations in genes encoding the RPE-secreted proteins TIMP3 (8) and EFEMP1 (9) cause lateronset macular disease.

Conflict of interest: The authors have declared that no conflict of interest exists. Citation for this article: J Clin Invest. 2011;121(1):369-383. doi:10.1172/JCI44303.
In addition to these specific RPE defects, histological evidence suggests that general RPE dysfunction and death play a primary, causative role in the pathogenesis of age-related macular degeneration (AMD) $(10,11)$. The atrophic form of AMD is characterized by subepithelial deposits, termed drusen, together with RPE and photoreceptor degeneration that involve the macula and that can extend into large areas of the posterior retina (12). A frequently proposed mechanism of RPE dysfunction in AMD posits accumulation of oxidative damage to RPE cell components, a consequent reduction in RPE mitochondrial function $(13,14)$, build up of subepithelial deposits, and inflammation at the RPE/choroid interface (15). The RPE resides in an oxygen-rich environment (16), and RPE mitochondrial DNA (mtDNA) is particularly prone to oxidative damage (13). The daily phagocytosis of photoreceptor outer segments by the RPE leads to the accumulation of the phototoxin $N$-retinyl- $N$-retinylidene ethanolamine (A2E) and other compounds that can inhibit mitochondrial function $(13,14,17)$, possibly through oxidative damage (18). mtDNA isolated from macular RPE of individuals with AMD exhibits significantly more damage than that from age-matched controls (19). Genetic studies implicate several mtDNA haplotypes $(20,21)$ and a nuclear gene encoding a mitochondria-associated protein (22) as AMD risk factors. Moreover, the RPE appears to be the initial site of pathogenesis in retinopathies associated with inherited mtDNA mutations (23), which can preferentially affect the macula $(24,25)$. Together, histological, biochemical, and genetic evidence suggests that damage to RPE mitochondrial function plays an important role in human retinal degenerative diseases. 
The cellular consequences of diminished mitochondrial function can be profound. Mitochondria integrate cellular stress responses that can lead to programmed cell death. Treatment of cultured RPE cells with A2E $(17,18)$ or oxidants $(26,27)$ causes mitochondrial membrane depolarization and release of proapoptotic mitochondrial proteins, such as cytochrome $c$ and apoptosis-inducing factor (AIF). Mitochondria also serve a vital role in energy metabolism. They house the cellular machinery for the tricarboxylic acid cycle and oxidative phosphorylation (OXPHOS), which together effectively convert glycolytic pyruvate to ATP and the by-products carbon dioxide and water. In the presence of oxygen, most differentiated cells primarily rely on mitochondrial OXPHOS to metabolize glucose, rather than aerobic glycolysis, an alternative cytoplasmic pathway used by proliferating cells to convert glucose to lactate, with a much lower molar yield of ATP (28). The RPE contains abundant mitochondria, consistent with a metabolically active tissue that performs a variety of energy intensive tasks (14), and RPE cells in culture exhibit a high degree of oxygen consumption (29). However, cultured RPE cells are adept at aerobic glycolysis (30) and are resistant to cell death in the face of OXPHOS inhibition, provided glucose is plentiful and glycolysis is not substantially compromised (31). Thus, the in vivo consequences of compromised RPE mitochondrial function leading to diminished OXPHOS are not obvious.

Despite the central role of the RPE in numerous retinal degenerative diseases, little is known about adult RPE stress responses in vivo. Many studies have described the responses of cultured primary or immortalized human RPE cells to a wide variety of perturbations, including growth factors, cytokines, hypoxia, and the aforementioned oxidative stress (32). However, cell culture studies do not model RPE tissue interactions with Bruch's membrane and fenestrated choroidal endothelium on the basal side and the intimate association of RPE microvilli and photoreceptor outer segments on the apical side. While more than 15 murine genetic models of AMD have been created, characterization has largely consisted of a description of morphological changes and comparison with those seen in humans with the disease (33). Few studies have assessed the molecular consequences of RPE stress in vivo $(34,35)$.

To characterize the in vivo response of the RPE to a reduction in OXPHOS, we generated mice with an RPE-selective postnatal loss of mtDNA transcription and replication. We reasoned that complete postnatal loss of RPE mitochondrial OXPHOS in the comparatively short-lived mouse might mimic changes caused by prolonged damage to RPE mtDNA and proteins in humans. Intriguingly, we found that RPE cells lacking OXPHOS are long lived in vivo and, over a period of months, undergo a series of morphological changes, many of which are reminiscent of those documented in human retinal degenerative disease. The mammalian target of rapamycin (mTOR) pathway is activated early in the process and drives dedifferentiation of the RPE through reduction in expression of RPE-characteristic proteins. Activation of $\mathrm{mTOR}$ and RPE dedifferentiation also occurs in response to a qualitatively different insult, chemical oxidative damage, suggesting a more general RPE stress response. Inhibiting this stress response by blocking mTOR activation has remarkable beneficial effects on the RPE and photoreceptors.

\section{Results}

Generation of RPEAMT mice. To generate mice with RPE-selective postnatal loss of OXPHOS (referred to herein as RPEAMT mice), we combined a conditional (floxed) allele of the nuclear-encod- ed gene for mitochondrial transcription factor A, Tfam, which is essential for mtDNA transcription and replication (36), with a cre transgene controlled by a fragment of the human BEST1 promoter known to drive RPE-specific expression in the eye (37). The BEST1-cre transgene drives ocular cre expression that begins postnatally, that is restricted to the RPE (Supplemental Figure 1A; supplemental material available online with this article; doi:10.1172/JCI44303DS1), and that does not cause cre toxicity in controls (Supplemental Figure 1, B and C). In both pigmented and

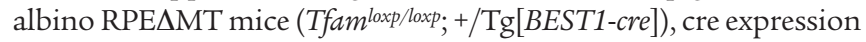
begins around P10 and rapidly increases to include more than $90 \%$ of all RPE cells by 9 weeks of age, with cre-mediated recombination detected only in the RPE and testis (38), as expected (6).

Diminished OXPHOS capacity and mitochondrial enlargement in RPE $\triangle M T$ RPE. Knockout of Tfam in cardiomyocytes (39), pancreatic $\beta$ cells (40), and cortico-hippocampal neurons (41) leads to loss of mtDNA and OXPHOS capacity, with kinetics that vary among the tissues. We found a conspicuous reduction in the level of mitochondrial-encoded cytochrome $c$ oxidase I (Cox1) mRNA in the RPE of 5-week-old RPE $\triangle \mathrm{MT}$ mice (Figure 1, A-D) and reduced levels of several other mitochondrial-encoded RNAs and Tfam mRNA in RPE cells isolated from 14-week-old RPEAMT mice (Figure 1E). We evaluated electron transport chain function in RPE $\triangle \mathrm{MT}$ mice by enzyme histochemical costaining for succinate dehydrogenase (SDH) and cytochrome c oxidase (COX, complex IV), only the latter of which contains mitochondrial-encoded catalytic subunits, and found COX enzymatic activity specifically diminished in a majority of RPE cells at 6 weeks of age (Figure 1, G and I). Consistent with findings in other TFAM-deficient tissues (36, 39, 40, 42), RPE $\mathrm{MT}$ RPE cells develop enlarged mitochondria with tubular cristae (Figure 1K), which can occupy as much as $70 \%$ of the cellular area in cross section (Figure 1L). The morphological changes in RPE mitochondria are accompanied by increases in the nuclear-encoded mitochondrial electron transport chain proteins, AIF, and cytochrome $c$ oxidase subunit IV (COX4) (Supplemental Figure 2, A and C), consistent with a futile compensatory response of increased mitochondrial biogenesis to diminished OXPHOS (43). These results demonstrate that postnatal loss of Tfam function in RPEAMT RPE cells causes a rapid, severe OXPHOS deficit and subsequent mitochondrial enlargement.

Minimal RPE cell death, autophagy, and senescence in RPEAMT mice. Accumulation of mtDNA mutations induces apoptosis (44), and increased cell death/loss is a prominent feature of other TFAMdeficient tissues $(41,42,45)$. Surprisingly, we found no evidence of caspase-3, -7 , or -9 activation above control levels in RPE $\Delta \mathrm{MT}$ mice at any age tested and as old as 1 year (data not shown). We also found little evidence of nuclear condensation or DNA fragmentation (Supplemental Figure 2E), and TUNEL, which detects both apoptotic and necrotic cells, revealed minimal RPE cell death in RPE $\triangle M T$ mice at all times tested (data not shown). Moreover, nuclear translocation of AIF, which is required for its proapoptotic role in caspase-independent apoptosis (46), was not remarkable in the RPE of RPE $\triangle M T$ mice at any age tested, although the level of cytoplasmic AIF was substantially increased (Supplemental Figure $2 \mathrm{E})$. We found no noticeable changes in macroautophagy markers (ATG12-ATG5 and LC3B), chaperone-mediated autophagy markers (LAMP-1 and -2), or senescence-associated $\beta$-galactosidase activity (47) in RPE $\triangle M T$ mice at any age tested (data not shown). Thus, RPE cells in vivo appear largely resistant to cell death, autophagy, and senescence under conditions of severe OXPHOS deficiency. 

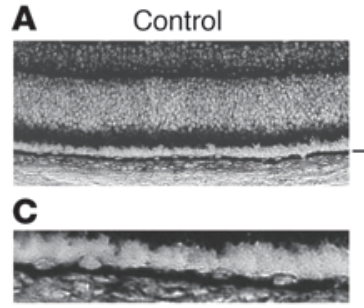

$\mathbf{F}$

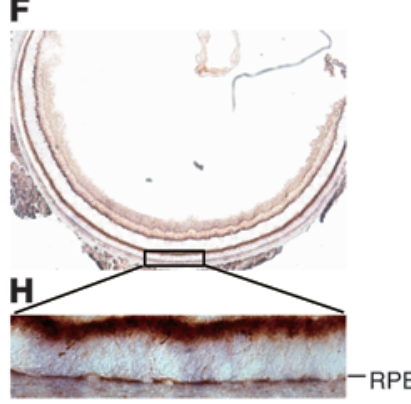

J

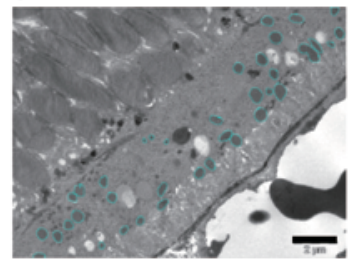

B RPE-

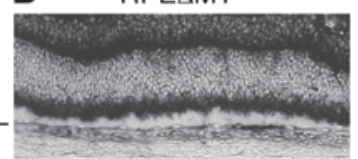

D

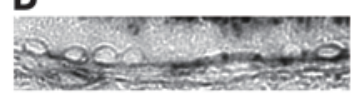

G

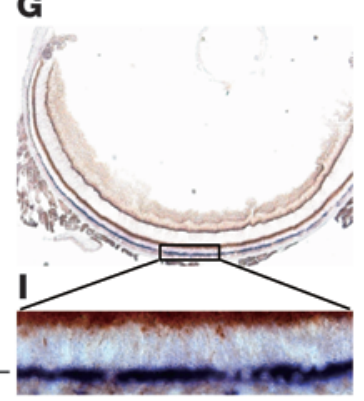

$\mathbf{K}$

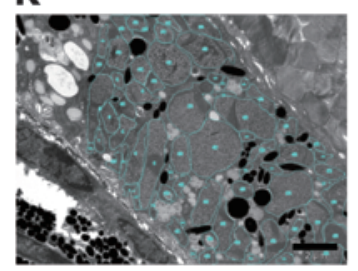

E

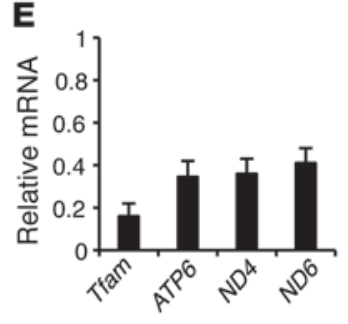

ened cells also had nuclear abnormalities, such as apical placement (Figure 2, E and F, and Supplemental Figure 3, B and D) and vertical alignment, in the case of binucleate cells (Supplemental Figure 3, B, D, and F). In cre-expressing RPE cells from pigmented mice, melanosomes were abnormally distributed after 14 weeks (Supplemental Figure 3, B and D), leading later to frequent areas of hypopigmentation and, occasionally, hyperpigmentation (Supplemental Figure 3J), which are attributes of human retinal degenerative disease (48). We did not observe by electron microscopy any examples of melanosome extrusion from RPE cells, although such profiles are admittedly rare in retinal degenerations.

The RPE hypertrophy and other abnormalities were accompanied by signs of RPE dedifferentiation. Ultrastructural analysis of RPE $\triangle M T$ mice at 22 weeks revealed loss of apical microvilli and basal infoldings (Figure 2, I and K, and Supplemental Figure $3 \mathrm{~L}$ ), key morphological indicators of RPE polarity. The abnormal localization of the RPE apical marker, ezrin (Supplemental Figure $3 \mathrm{H}$ ), confirmed depolarization of cre-expressing RPE cells. Moreover, the levels of 5 out of 6 RPE-characteristic proteins tested were significantly reduced at 14 weeks of age and more so by 22 weeks (Figure 2, L and M). mRNA levels for these markers were also reduced (Supplemental Figure 3Q). RPE65, a marker of mature RPE, exhibited the greatest reduction at any age tested (Figure 2M), and diminished levels of Rpe 65 mRNA and protein were evident as early as 6 and 10 weeks of age (Supplemental Figure 3, N and P), closely tracking depletion of mtRNA. Significantly, of the 6 RPE-characteristic markers tested, only MITF, a transcription factor associated with determination of RPE cell fate during development (49), was increased in expression (Figure 2, L and M).

OXPHOS deficiency induces RPE dedifferentiation and bypertrophy. The long-term viability of RPE cells in RPE $\triangle M T$ mice allowed us to characterize their morphological and molecular changes and assess similarities to human retinal degenerative diseases. RPE flat mounts ( $x, y$ plane) at 16 weeks revealed a loss of regular cuboidal appearance and an increase in heterogeneity of size and shape of cre-expressing cells (Figure $2 \mathrm{~B}$ ) compared with those of controls (Figure 2A). On cross section ( $z$ plane), thickened ( $>5 \mu \mathrm{m}$ ) RPE cells are frequently seen as early as $12-14$ weeks (Figure 2D), and these cells express cre (Supplemental Figure 3, B and D). By 26 weeks, a majority of RPE cells were uniformly thickened (Figure 2E), indicating a similar morphological change in all cre-expressing RPE cells, which account for approximately $85 \%$ of the total at this time. Maximum RPE thickness in RPEAMT mice increased to $15 \pm 3.3$ $\mu \mathrm{m}$ by 35 weeks, 3 times that of controls (Figure 2, F and G). Thick-
Cellular proliferation can accompany dedifferentiation of otherwise postmitotic tissues, including RPE (50). We therefore assessed RPE proliferation by BrdU labeling at various ages. Although the numbers were not large, BrdU-reactive RPE cells in RPE $\triangle M T$ mice at 18 and 26 weeks of age were significantly more numerous than in controls (Figure $2 \mathrm{~N}$ ) and included both creexpressing and cre-negative cells (Figure 2, P and Q). At 3 months after injection (PI), cells with multiple BrdU-reactive nuclei were still present (Figure 2, P and Q), indicating that some proliferating cells were unable to accomplish cytokinesis, a feature also seen in human RPE surrounding drusen (51). Despite this pathological proliferation, RPEAMT mice exhibited a gradual loss of RPE cells over time (Figure 2O). Together, the morphological abnormalities, the loss of markers of mature RPE, and the increase in expression of an RPE cell fate determinant in RPE $\triangle M T$ mice indicate a 

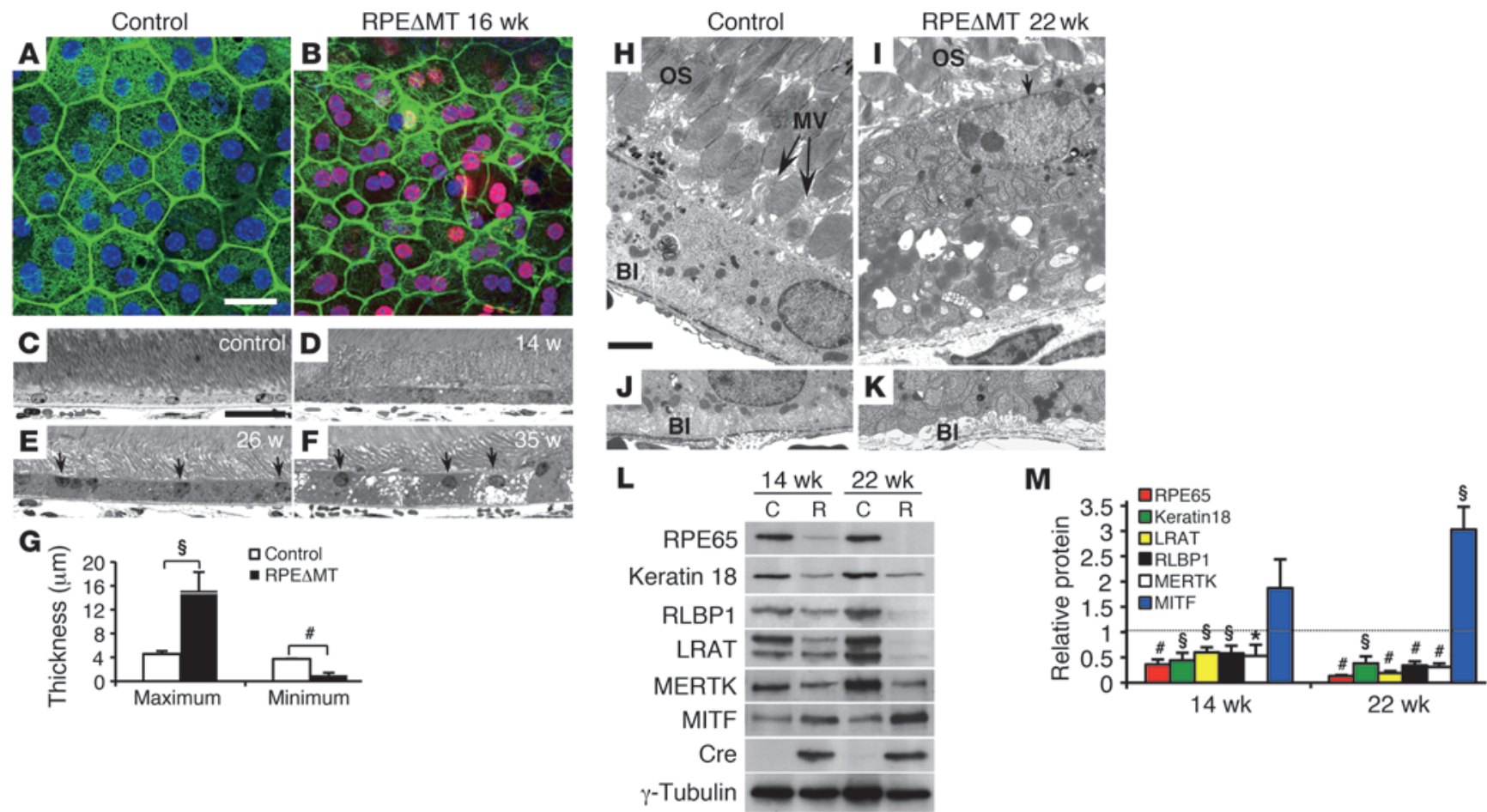

$\mathbf{N}$
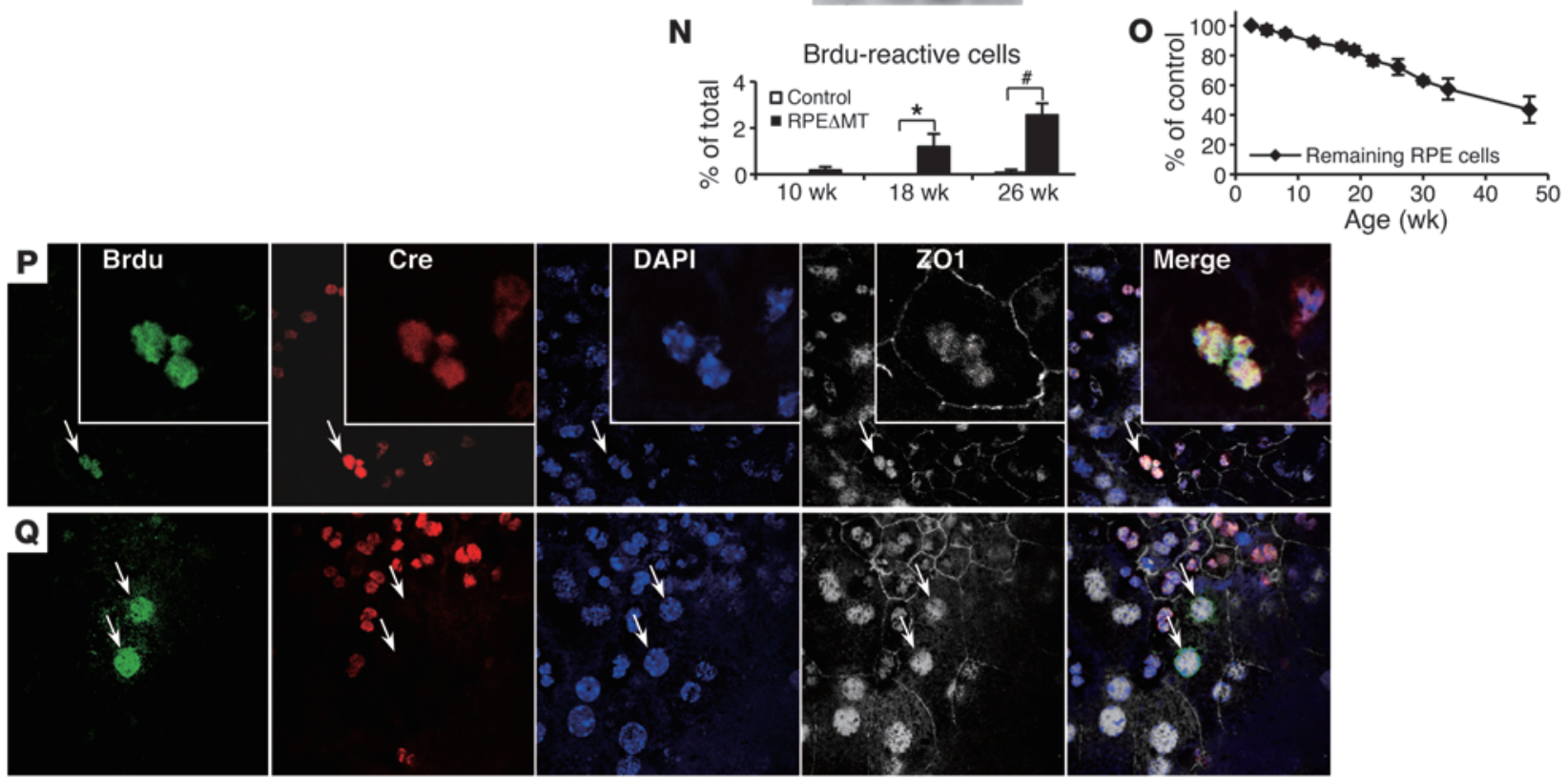

\section{Figure 2}

RPE dedifferentiation and hypertrophy in RPE $\triangle$ MT mice. (A and B) RPE flat mounts stained for cre (red) and phalloidin (green) show (B) loss of cuboidal appearance in cre-reactive cells in RPE $\Delta M T$ mice at 16 weeks of age. (C-F) Light microscopy reveals thickened RPE cells (D) in a 14-week-old RPE $\Delta$ MT mouse. (E and F) Numerous RPE cells of RPE $\Delta M T$ mice have progressively increased thickness and apical location of nuclei (arrows). (G) At 35 weeks of age, both maximum and minimum RPE thickness are significantly different between RPE $\Delta M T$ mice and controls. (H-K) Electron microscopy in 22-week-old RPE $\Delta \mathrm{MT}$ mice (I and $\mathbf{K}$ ) shows a thickened RPE cell, with apical location of nucleus, (I, arrow denotes nucleus, OS stands for outer segments) loss of microvilli (MV), (H) arrows denote MV, and (K) absent basal infoldings (BI). (L) Loss of a series of RPE-characteristic markers, with the exception of MITF, in RPE cells from RPE $\Delta M T$ mice (R) versus control (C) is detected by immunoblot, and (M) confirmed by quantification of protein. $(\mathbf{N})$ The percentage of BrdU reactive RPE cells at 2 days PI is significantly increased in RPE $\triangle$ MT mice at 18 weeks and older. (0) However, RPE $\triangle$ MT mice have a gradual loss of RPE cells over time. (P and $\mathbf{Q})$ BrdU staining shows cre-expressing ( $\mathbf{P}$, arrows denote; insets are pictures of the same cell with higher magnification) and cre-negative RPE cells ( $\mathbf{Q}$, arrows) that have multiple BrdU-reactive nuclei within 1 cell boundary (denoted by ZO1) at 3 months PI. Original magnification, $\times 400$. Error bars in G, M, N, and $\mathbf{O}$ represent SD from triplicates. Scale bars: $25 \mu \mathrm{m}(\mathbf{A}-\mathbf{F}) ; 2 \mu \mathrm{m}(\mathbf{H}-\mathbf{K})$. ${ }^{\star} P<0.05 ;{ }^{\S} P<0.01$; ${ }^{*} P<0.001$. 

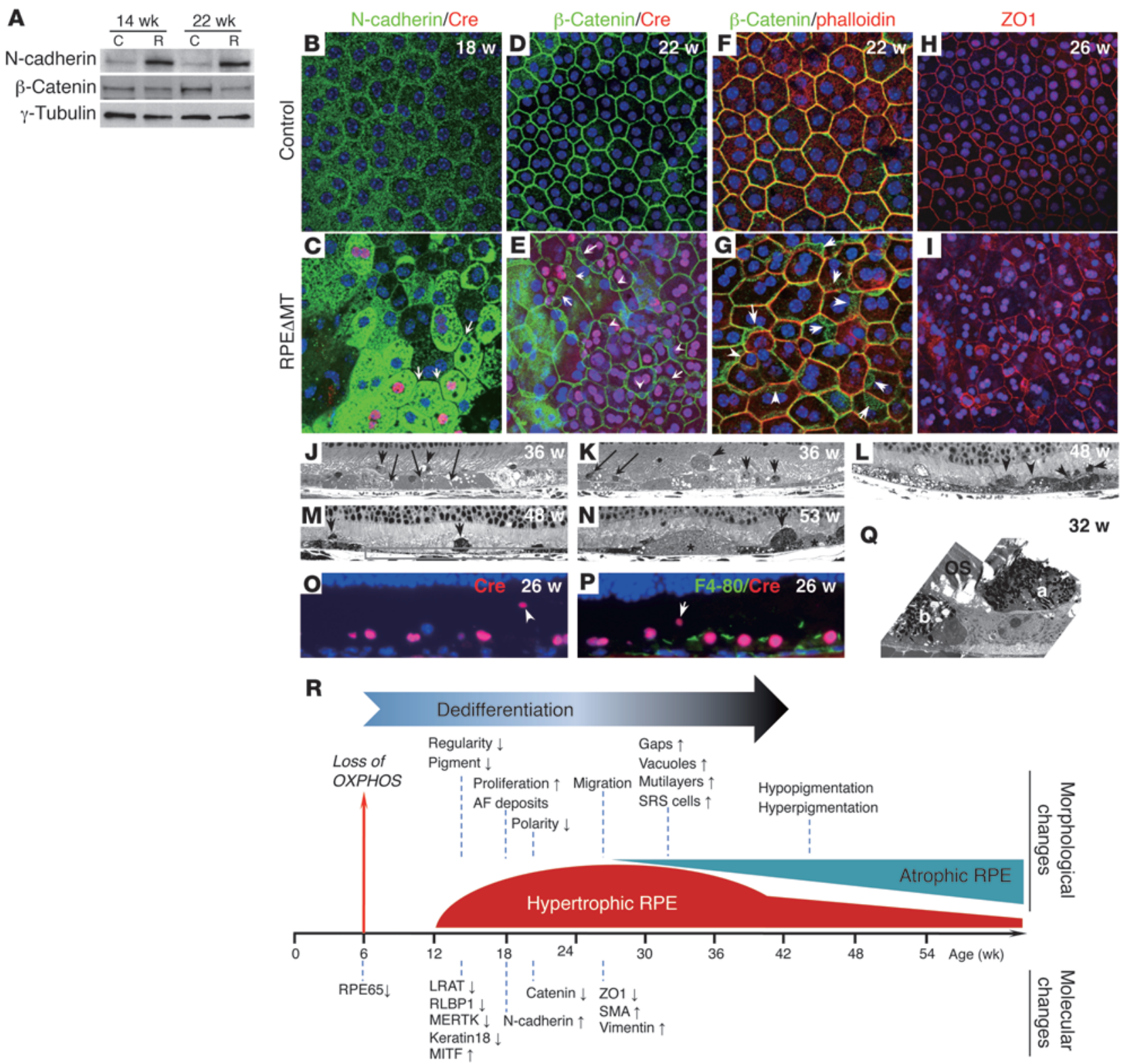

\section{Figure 3}

Decreased junctional integrity and increased migration of RPE cells in RPE $\triangle \mathrm{MT}$ mice. (A) Immunoblot of RPE cells from pigmented RPE $\triangle \mathrm{MT}$ mice shows increased N-cadherin and reduced $\beta$-catenin. (B-I) Immunostaining of RPE flat mounts from RPE $\Delta M T$ mice (C, E, G, and I) shows $\mathrm{N}$-cadherin (green) substantially increased in RPE cytoplasm but diminished at cell junctions (C, arrows denote cell junctions). $\beta$-Catenin staining (green) is obscured at cre-expressing cell (red) boundaries, with a cytoplasmic redistribution (E, arrows), which is confirmed by co-labeling of $\beta$-catenin (green) and phalloidin (red) (G, arrows). (I) A stacked Z-series confocal image (15-um thickness) shows diminished ZO1 staining (red) at RPE cell junctions. Original magnification, $\times 400$. (J-N) Light microscopy of albino ( $\mathbf{J}$ and $\mathbf{K})$ and pigmented $(\mathbf{L}-\mathbf{N})$ RPE $\Delta M T$ mice demonstrates gaps between RPE cells ( $\mathbf{J}$, arrows), RPE vacuoles ( $\mathbf{J}-\mathbf{L})$, multilayered RPE cells (K, arrows), and numerous cells in the subretinal space (arrowheads in $\mathbf{J}-\mathbf{N}$ ), which leads to an uneven RPE appearance at late stages as well as RPE atrophy (boxed area in $\mathbf{M}$ ). (N) Asterisks mark drusen-like material. ( $\mathbf{O}$ and $\mathbf{P}$ ) Cre-expressing RPE cells (red) are present in the subretinal space (arrowheads), whereas F4-80 reactivity (green), indicative of macrophages/microglia, is restricted to less than $2 \%$ of the RPE layer at 26 weeks. (J-P) Original magnification, $\times 630$. (Q) An electron micrograph from an RPE $\Delta M T$ mouse shows 2 pigmented cells of similar appearance ( $a$ and $b$ ), 1 of which a has migrated to the subretinal space. Original magnification, $\times 5000$. (R) Loss of OXPHOS causes a sequence of morphological and molecular changes, beginning with a process of RPE dedifferentiation. Up and down arrows denote increased and decreased expression, repectively.

process of RPE dedifferentiation that is an early consequence of mitochondrial dysfunction.

Decreased RPE junctional integrity and increased cell migration cause widespread atrophy. We investigated the mechanism underlying the gradual loss of RPE cells in RPE $\triangle \mathrm{MT}$ mice by assessing adherens and tight junctions as measures of epithelial integrity. By 18 weeks of age, $\mathrm{N}$-cadherin, the dominant cadherin isoform in the RPE adherens complex (32), was markedly increased (Figure 3, A 
and C), but in most cre-expressing cells the protein was primarily cytoplasmic rather than at cell membranes (Figure 3C and Supplemental Figure 4B). Similar to $\mathrm{N}$-cadherin, $\beta$-catenin exhibited increased cytoplasmic (Figure 3E and Supplemental Figure 4D) and decreased cell boundary (Figure 3G) localization in cre-expressing cells. Despite a reduction in total $\beta$-catenin levels at 22 weeks (Figure 3A), phosphorylation of $\beta$-catenin at $\mathrm{Y} 654$ and $\mathrm{Y} 489$, which triggers disassembly of the $\mathrm{N}$-cadherin/ $\beta$-catenin adherens complex (52), increased in RPE $\triangle M T$ mice with age (Supplemental Figure 4I). Consistent with disruption of adherens junctions, nuclear and cytoplasmic PY489- $\beta$-catenin increased in RPEAMT RPE (Supplemental Figure 4, K and M) relative to a control (Supplemental Figure 4, J and L). We also observed disruption of RPE tight junctions in RPE $\triangle \mathrm{MT}$ mice as early as 26 weeks of age, as evidenced by diminished ZO-1 abundance at many cell boundaries (Figure 3I).

Disruption of adherens and tight junctional complexes in RPE $\triangle M T$ mice caused changes similar to those seen in some human retinal degenerative diseases, such as AMD. By 32 weeks of age, gaps between RPE cells were evident (Figure 3J) and numerous cells were present in the subretinal space (Figure 3, J-L). In pigmented RPEAMT mice, most subretinal cells contained melanosomes (Figure 3, L-N and Q), suggesting that they are either displaced RPE cells or macrophages that have ingested pigment. The presence of cre (Figure 3O), but not a macrophage/microglia marker (Figure 3P), in the subretinal cells conclusively established their RPE origin. RPE cell migration, which may explain the gradual RPE cell loss we observed (Figure 2O), eventually results in extraordinary variation in thickness of the epithelium, from multilayered to highly attenuated (Figure 3, J-M). Some thickened cells contained prominent vacuoles (Figure 3, J-L), and drusen-like material was present in mice older than 1 year (Figure $3 \mathrm{~N}$ ). Taken together, loss of junctional integrity and increased cell migration indicate the beginning of an epithelial-to-mesenchymal transition (EMT) in RPEAMT RPE. Indeed, the changes in cadherin and $\beta$-catenin that we described are hallmarks of EMT (32) and are consistent with an observed increase in the mesenchymal markers smooth muscle actin and vimentin at 26 weeks (Supplemental Figure 4, E-H). Thus, ablation of RPE mitochondrial OXPHOS initiated a sequence of molecular and morphological changes that result in early dedifferentiation and hypertrophy and, later, EMTlike changes and widespread atrophy (Figure 3R).

Progressive photoreceptor degeneration in RPEAMT mice. In the atrophic form of advanced AMD, photoreceptors are thought to degenerate as a consequence of RPE cell dysfunction and death. We monitored the response of the neural retina to RPE dedifferentiation in RPEAMT mice by histological and electrophysiological methods. Regions of photoreceptor loss adjacent to hypertrophic RPE were evident histologically as early as $14-16$ weeks of age (e.g., Figure 4A and Supplemental Figure 5A), whereas a global deficit in photoreceptors was not apparent prior to 26 weeks. By 36 weeks of age, albino RPE $\triangle \mathrm{MT}$ mice lost approximately $18 \%$ of all photoreceptors (Figure 4, B and C) and, by 56 weeks of age and older, pigmented mice lost approximately $34 \%$ of photoreceptors (Supplemental Figure 5, B and C). At 40 weeks, but not at 20 weeks, numerous macrophages/microglia had invaded the subretinal space (Supplemental Figure $4 \mathrm{~N}$ ), as is commonly seen in later stages of photoreceptor degenerations in mice. As early as 22 weeks of age, cones exhibited severely shortened morphology and lower density (Figure 4, E and G), particularly in the posterior ventral retina and always in association with abnormal RPE.
Notably, the number of RPE cells was not severely decreased at this age (Figure 2O). Global quantification by light microscopy revealed that only approximately $49 \%$ of total cones ( $~ 55 \%$ dorsal, $\sim 43 \%$ ventral) remained at 36 weeks, an age at which approximately $82 \%$ of total photoreceptors, $97 \%$ of which are rods, were still present (Figure 4C). While cone morphology and survival are preferentially affected by RPE dedifferentiation, both scotopic and photopic electroretinographic (ERG) responses were reduced (Figure 4H and Supplemental Figure 5D). These effects correlated with the striking reduction in RPE65 that is an early consequence of loss of OXPHOS (e.g., Figure 4E). Thus, RPE dedifferentiation degrades both rod and cone function relatively rapidly and results in an early, preferential, and regional loss of cones.

RPE $M$ M RPE cell survival via enhanced glycolytic flux and HGF/ $c$-Met pathway activation. To understand the mechanisms underlying the morphological and behavioral changes of RPE cells induced by loss of OXPHOS, we evaluated selected metabolic and signal transduction pathways. RPE cells in culture exhibit a large "Pasteur effect," meaning that they can efficiently metabolize glucose to lactate via aerobic glycolysis when mitochondrial OXPHOS is blocked (31). We therefore assessed glycolytic flux in RPEAMT mice. We measured lactate secretion and intracellular glycerol 3-phosphate (G3P) and NAD levels as metabolic readouts of glycolytic flux and found that levels in RPE $\triangle M T$ RPE cells of all 3 metabolites are significantly increased, several hundred-fold for G3P and NAD (Supplemental Figure 6, A and B). Also, consistent with a metabolic shift to aerobic glycolysis, GLUT1 was increased in RPE $\triangle M T$ RPE cells (Supplemental Figure 6, D-F). Importantly, ATP levels remained similar between RPEAMT and control RPE cells (Supplemental Figure 6C), indicating that increased glycolytic flux can largely compensate for OXPHOS deficiency, at least with respect to ATP generation. This compensatory metabolic shift in the RPE is similar to the Warburg effect in cancer cells: a switch from OXPHOS to aerobic glycolysis to increase the rate of ATP and biomass production (53).

Increased aerobic glycolysis in normal cells requires protein factors that also promote cell growth (28) and, often, EMT-like changes (54). We therefore evaluated a series of growth factors and/or pathways in RPEAMT RPE cells as detailed in Supplemental Tables 1 and 2. Among them, we found a substantial elevation in hepatocyte growth factor (HGF) at both the mRNA and protein levels (Figure 5A and Supplemental Figure 7, A-D). We also detected activation of the HGF receptor, c-Met, as evidenced by phosphorylation at Y1234/1235 (Figure 5A), which is required for tyrosine kinase activity (55). Immunostaining of retinal sections confirmed activation of HGF/c-Met in the RPE (Figure 5, C and E), particularly in cre-expressing cells (Supplemental Figure 7, D and $\mathrm{F}$ ), supporting an autocrine mechanism.

HGF has an important role in activating glycolysis (56) and blocking apoptosis $(26,27)$. We reasoned that HGF/c-Met activation may also be required for RPE cell survival in RPE $\triangle \mathrm{MT}$ mice. To test this hypothesis, we treated RPE $\Delta$ MT mice ( 26 weeks old) for 6 days with a selective c-Met inhibitor, PHA-665752 (55), or vehicle ( $n=6$ for each group). Control mice were also administrated PHA-665752 or vehicle to assess the effect of the inhibitor on normal RPE. At 2 days PI, PHA-665752 remarkably reduced phosphorylation of c-Met ${ }^{\mathrm{Y} 1234 / 1235}$ in RPE $\Delta \mathrm{MT}$ RPE cells (Figure $5 F$ ). Inhibition of c-Met did not affect the RPE of controls (Supplemental Figure 7, G and H). However, RPE $\Delta \mathrm{MT}$ mice treated with PHA- 665752 but not vehicle had numerous caspase-9-positive 

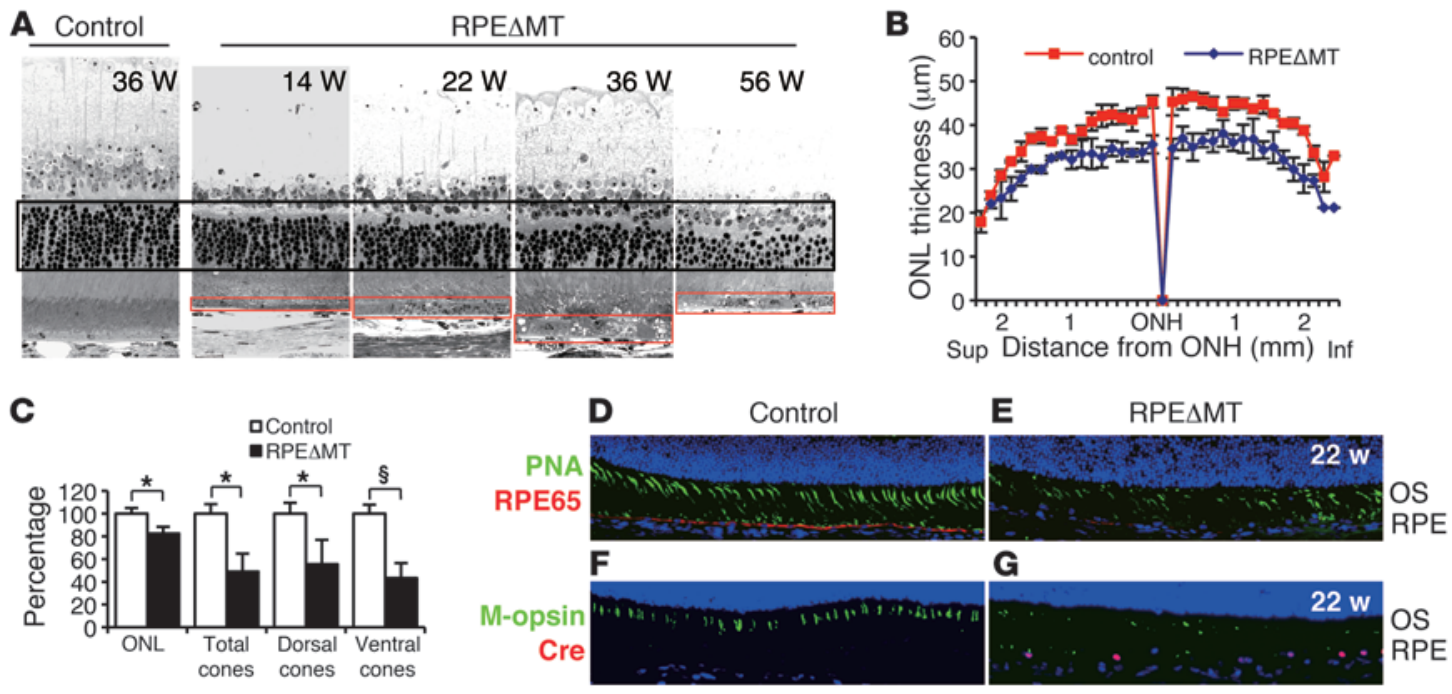

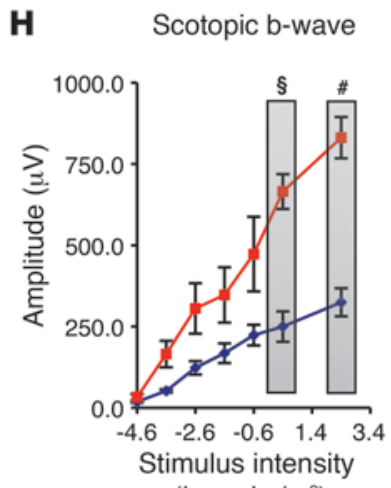

$\left(\log \mathrm{cd} \mathrm{s} / \mathrm{m}^{2}\right)$

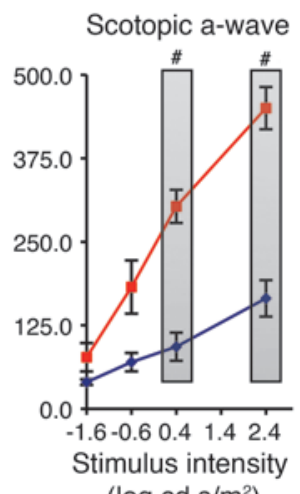

$\left(\log \mathrm{cd} \mathrm{s} / \mathrm{m}^{2}\right)$

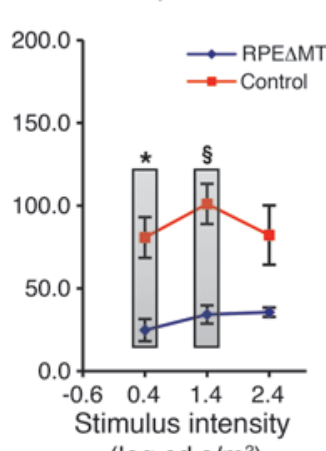

$\left(\log \mathrm{cd} \mathrm{s} / \mathrm{m}^{2}\right)$

Figure 4

Photoreceptor degeneration correlates with RPE dedifferentiation in albino RPE $\Delta \mathrm{MT}$ mice. (A) Light micrographs of the posterior retina show a progressive regional loss of photoreceptors reflected by outer nuclear layer (ONL) thickness (black box) and correlating with RPE dedifferentiation (red boxes). Original magnification, $\times 630$. (B) Global assessment of outer nuclear layer thickness in 36-week-old RPE $\Delta$ MT mice $(n=3)$ and controls $(n=3)$. Sup., superior retina; ONH, optic nerve head; Inf., inferior retina. (C) Quantification of mean outer nuclear layer thickness and cones shows a significant loss of rod and cone photoreceptors in 36-week-old RPE $\Delta$ MT mice $(n=3)$ compared with that in controls $(n=3$, mean value of controls is defined as 100\%). (D-G) Immunostaining images from the posterior ventral retinas of 22-week-old mice. Original magnification, $\times 200$. (D and E) Costaining for lectin-PNA (green) and RPE65 (red) demonstrates (E) a striking loss of cones in RPE $\Delta M T$ mice, which correlates with diminished RPE65 protein. (F and G) Immunostaining for M-opsin (green) and cre (red) shows abnormalities of red/green opsin-expressing cones, which correlate with cre-expresssing cells $(\mathbf{G})$. (H) Electroretinograph demonstrates significantly reduced rod responses (scotopic a-wave and b-wave) and cone responses (photopic b-wave) in 36-week-old RPE $\Delta$ MT mice $(n=3)$, compared with those in controls $(n=5)$. Verticle bars clarify the 2 groups of values used for statistical comparison. Data in $\mathbf{H}$ represent mean \pm SEM. ${ }^{*} P<0.05 ; \$ P<0.01 ;{ }^{\sharp} P<0.001$.

apoptotic RPE cells with reduced cre expression and a shrunken appearance at PI 2 day (Figure 5I). Furthermore, stacked confocal images of flat mounts from PHA-665752-treated RPE $\Delta$ MT mice showed large areas with faint or absent phalloidin staining and few to no cre-expressing RPE cells (Figure 5K), indicating that many apoptotic cells were rapidly lost by PI 2 day. Similarly, retinal sections from PHA-665752-treated RPEAMT mice reveal substantial segments of Bruch's membrane that appeared largely denuded of RPE cells (Figure 5M). As a result of these changes, which were completely absent in either vehicle-treated or untreated RPE $\triangle M T$ mice (Figure 5, H, J, and L), the number of remaining RPE cells was significantly decreased in RPEAMT mice treated with PHA-665752 (Figure 5G). Thus, activation of the HGF/c-Met pathway is crucial for survival of RPE cells deprived of OXPHOS.
Activation of PI3K/AKT/mTOR pathway and biomass accumulation explains RPE $\triangle M T$ RPE bypertrophy. The PI3K/AKT/mTOR pathway is frequently engaged in cells responding to growth factors, including HGF. We detected increased phosphorylation of PI3K $\left(\mathrm{p}^{\mathrm{Ty}} 5^{\mathrm{Tr} 458}\right.$ ) and $\mathrm{AKT}{ }^{\mathrm{Ser} 473}$ in RPE$\Delta \mathrm{MT}$ eyecups at 9 weeks (Supplemental Figure 8A) as well as in RPE cells isolated at 14 weeks (Figure $6 \mathrm{~A}$ ). Phosphorylation of glycogen synthase kinase-3 $\beta$ (GSK-3 $\beta$ ) at Ser9, a site known to be regulated by AKT, was also increased in RPE $\triangle \mathrm{MT}$ mice (Figure 6A), implying decreased GSK-3 $\beta$ activity (57). This is consistent with the observed increase in cytoplasmic and nuclear PY489- $\beta$-catenin (Supplemental Figure 4, K and M), because GSK-3 $\beta$ is an important trigger for $\beta$-catenin ubiquitination and subsequent degradation (58). PI3K/AKT pathway activation and decreased GSK-3 $\beta$ activity can also enhance glycolytic flux 

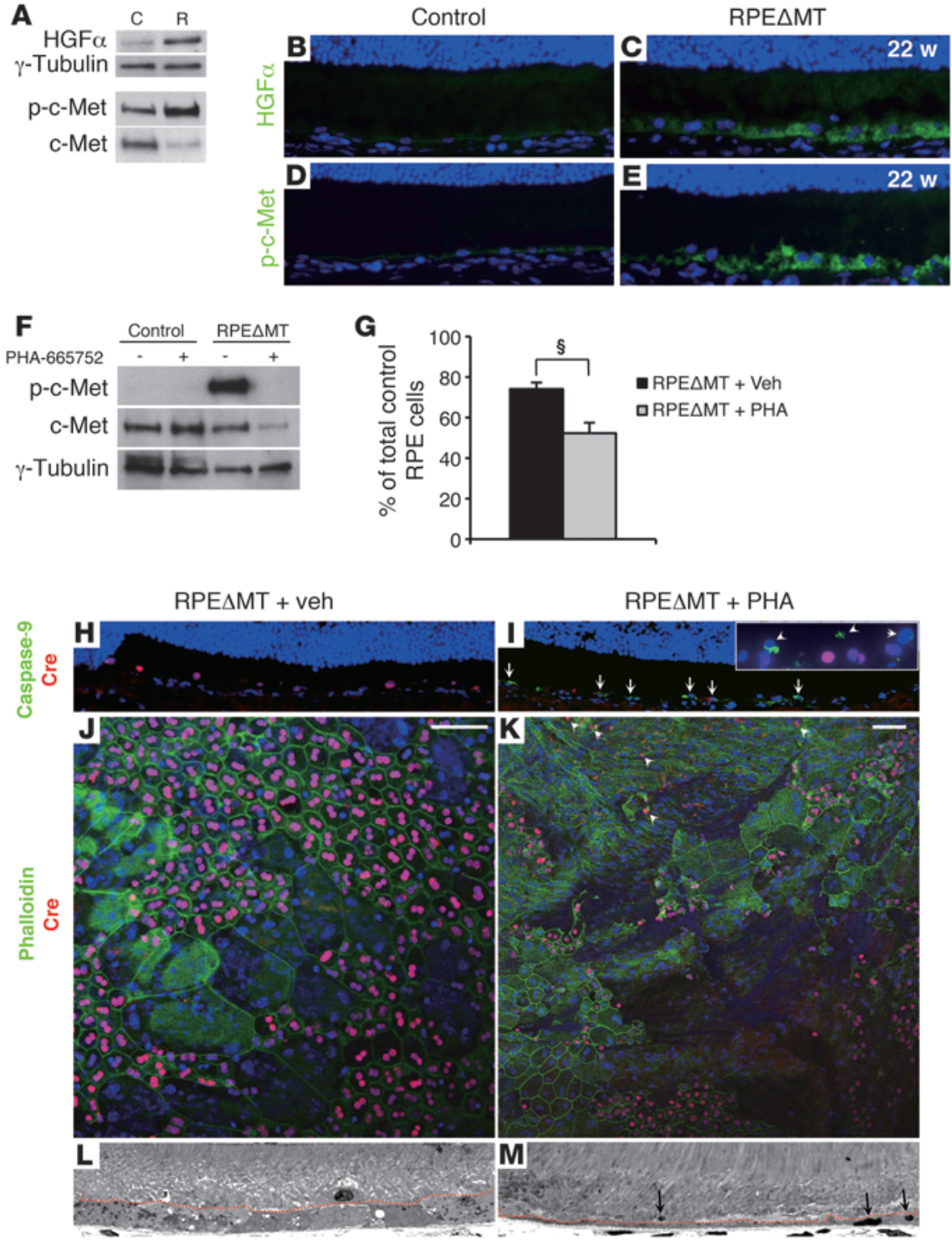

\section{Figure 5}

Activation of the HGF/c-Met pathway in RPE $\triangle M T$ mice is critical for RPE cell survival. (A) Immunoblot detects increased HGF and phosphorylated c-Met (Y1234/1235) in RPE cells from pigmented RPE $\Delta \mathrm{MT}$ mice at 14 weeks, compared with that in control. (B-E) Original magnification, $\times 400$. Immunostaining of retinal sections shows increased reactivity of HGF (C) and p-c-Met (Y1234/1235) (E) in 22-week-old albino RPEAMT mice. (F) Immunoblot shows diminished p-c-Met (Y1234/1235) reactivity in 26-week-old albino RPE $\Delta$ MT mice at 2 days $\mathrm{PI}$ of PHA-665752, a selective c-Met inhibitor. (G) Quantification of total remaining RPE cells at 2 days PI (normalized by the number of cells in vehicle-injected Tfam ${ }^{\text {loxplloxp }}$ control mice) reveals a significant loss of RPE cells in RPE $\triangle$ MT mice treated with PHA-665752 (PHA) $(n=3)$ versus RPE$\Delta$ MT mice treated with vehicle (Veh) $(n=3) . \S P<0.01$. ( $\mathbf{H}$ and $\mathbf{I})$ Immunostaining detects activated caspase-9reactive RPE cells (green) in PHA-665752injected RPE $\triangle M T$ mice (I, arrows; inset is higher magnification image from another area). ( $\mathbf{J}$ and $\mathbf{K}$ ) Staining for phalloidin and cre on RPE flat mounts. (K) Z-scanning images $(20-\mu \mathrm{m}$ stack) show large areas of atrophic RPE with attenuated phalloidin staining and few, condensed cre-expressing RPE cells (arrows) in PHA-665752-treated RPE $\Delta M T$ mice. (J) No effect is observed in vehicle-treated RPE $\triangle M T$ mice. $(\mathbf{H}-\mathbf{K})$ Original magnification, $\times 200$. ( $L$ and $M$ ) Light microscopy shows a denuded Bruch's membrane and tiny pyknotic RPE cells (arrows) in PHA-665752-treated RPEAMT mice (M), whereas RPE remains thickened and intact in vehicle-treated RPE $\triangle \mathrm{MT}$ mice (L). Dotted red lines in $\mathbf{L}$ and $\mathbf{M}$ denote the approximate edge of the outer segment layer. Original magnification, $\times 630$.
(28), which, together with increased phosphorylation of BAD $\mathrm{Ber}^{\mathrm{S} 136}$ (Supplemental Figure 8A), provide evidence for a PI3K/AKT-regulated cell survival mechanism in RPEAMT RPE (59). mTOR complex 1 (mTORC1) can be activated directly by AKT (60) or indirectly through inactivation of tuberous sclerosis complex-2 (TSC2) (61). mTORC1 in turn promotes cell growth by activating the ribosomal protein S6 kinase, $70 \mathrm{kDa} / \mathrm{S} 6$ ribosomal protein (P70S6K/S6) pathway (61). In RPE $\triangle M T R P E$ cells, we detected augmented phosphorylation of $\mathrm{mTOR}^{\mathrm{Ser} 2448}$, TSC2 ${ }^{\mathrm{Thr} 1462}$ (Figure 6, B and C), and the S6K substrates P70SK6 $6^{\text {Thr389 }}$ and S6 $6^{\text {Ser235/236 }}$ (Figure 6D and Supplemental Figure 8B), indicating enhanced activity of the mTORC1/S6K pathway. Immunostaining of frozen sections and/or flat mounts confirmed elevated phosphorylation of $\mathrm{AKT}^{\mathrm{Ser} 473}, \mathrm{mTOR}^{\mathrm{Ser} 2448}$, and

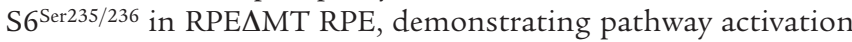
specifically within cre-expressing cells (Figure 6, F, H, J, and L, and Supplemental Figure 8, D, F, and H). Consistent with the known ability of the mTOR/S6K pathway to boost protein synthesis (61), protein content per RPE cell in RPE $\triangle \mathrm{MT}$ mice was significantly increased at 14 weeks of age as well as later (Figure $6 \mathrm{M}$ ).
$\mathrm{PI} 3 \mathrm{~K} / \mathrm{AKT} / \mathrm{mTORC} 1$ activation can stimulate lipogenesis in a variety of cell types (62), including immortalized RPE (63), and lipid accounts for $40 \%$ of drusen by weight (64). We detected a striking increase in the amount of neutral lipids in RPE $\triangle \mathrm{MT}$ mice at 14 weeks of age (Figure 6O). At 16 weeks, autofluorescent deposits were visible over a broad range of excitation wavelengths but bleached rapidly at $350 \mathrm{~nm}$ (Figure 6P). A Z-stack of the flat mount images in Figure 6P shows that the deposits had a lipid-like appearance and were clearly located within RPE cells (Figure 6Q). RPE lipid-like deposits were also evident by electron microscopy of retinal sections (Figure 6R). By 18 weeks and later, the fundi of RPEAMT mice exhibited prominent white spots (Figure 6S), likely a macroscopic manifestation of lipid buildup. Thus, activation of the PI3K/AKT/ mTOR pathway in RPE $\triangle$ MT RPE cells precedes most of the morphological changes that we observed (Figure 3R). Activation of this pathway provides a plausible mechanism for increased glycolytic flux, growth factor-dependent survival, and cellular accumulation of protein and lipid. The early onset of pathway activation indicates an initial response of the RPE to metabolic stress. 

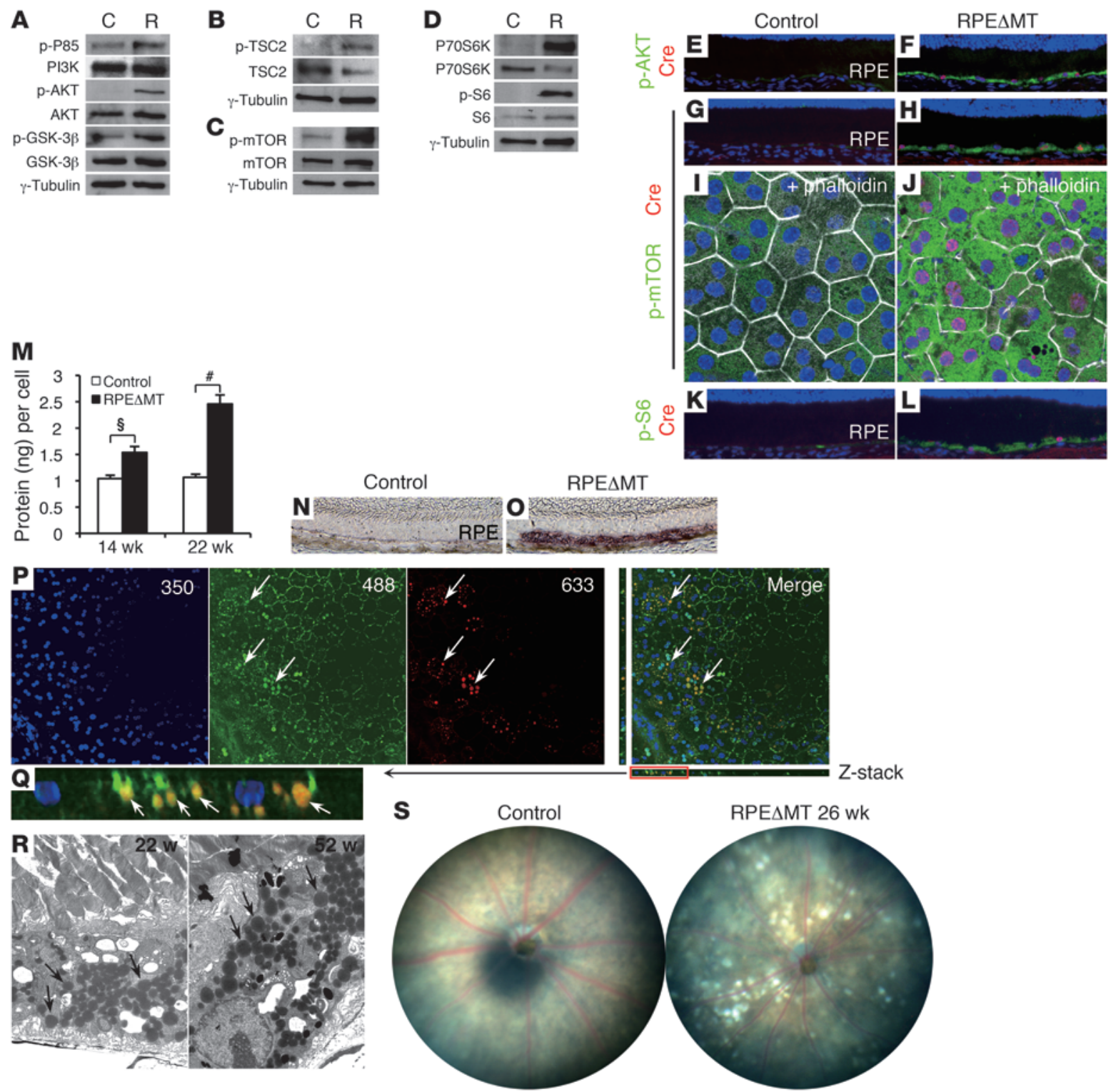

Figure 6

Activation of the PI3K/AKT/mTOR pathway and increased biomass in RPE $\triangle$ MT RPE. (A-D) Immunoblot detects elevated phosphorylation of

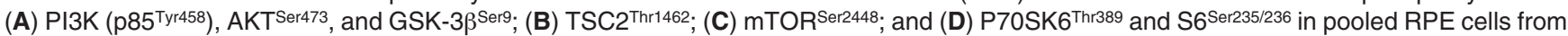
pigmented RPE $\triangle \mathrm{MT}$ mice at 14 weeks, compared with that in controls. $(\mathbf{E}-\mathbf{H})$ Original magnification, $\times 200$. (E-L) Immunostaining of retinal sections (E-H, K, and $\mathbf{L}$ ) and RPE flat mounts (I and $\mathbf{J})$ shows increased reactivity of phosphorylated AKTSer473 (F, green), mTORSer2448 $(\mathbf{H}$ and $\mathbf{J}$, green), and S6 $6^{\operatorname{Ser} 235 / 236}$ (L, green) in cre-expressing RPE cells (red/purple) in albino RPE $\Delta \mathrm{MT}$ mice. Phalloidin staining of RPE flat mounts (I and $\mathbf{J}$, white) outlines cell boundaries. Original magnification, $\times 630$. ( $\mathbf{K}$ and $\mathbf{L})$ Original magnification, $\times 200$. (M) Protein content is increased in RPE cells of RPE $\triangle M T$ mice (triplicate assays). ${ }^{\S} P<0.01$; ${ }^{*} P<0.001$. ( $\mathrm{N}$ and $\mathbf{O}$ ) Oil red $\mathrm{O}$ staining detects a remarkable accumulation of neutral lipid in 14-week-old RPE $\triangle$ MT RPE (O, purple), compared with that of a control (N). (P) Confocal microscopy of an RPE $\Delta M T$ flat mount reveals autofluorescent deposits (arrows) visible over a broad spectrum of excitation wavelengths (e.g., 350, 488, and $633 \mathrm{~nm}$ ). The deposits are initially visible at $350 \mathrm{~nm}$ but bleach rapidly, leading to their orange appearance in the merged picture (arrows). The flat mount was stained with anti$\beta$-catenin (green), which highlights RPE cell boundaries at $488 \mathrm{~nm}$. (Q) A higher magnification Z-stack image of the red boxed area in $\mathbf{P}$. Note that autofluorescent deposits are below $\beta$-catenin (green) and therefore inside RPE cells. (P and $\mathbf{Q})$ Original magnification, $\times 200$. (R) Electron micrographs of RPE $\triangle$ MT RPE reveal abundant structures consistent with lipid-containing granules (arrows). Original magnification, $\times 5000$. (S) Funduscopy shows white deposits in a pigmented RPEAMT mouse. Original magnification, $\times 50$.

Rapamycin preserves RPE differentiation features and photoreceptors in $R P E \triangle M T$ mice. In order to determine which of the RPE morphological and molecular changes derive from mTORC1 activation, we treated RPE $\triangle \mathrm{MT}$ mice daily with the MTORC1 inhibitor, rapa- mycin $(n=15)$, or vehicle $(n=15)$, beginning at 4 weeks of age. Littermate controls also received rapamycin $(n=14)$ or vehicle $(n=20)$ to assess the effect on normal RPE and photoreceptors. Rapamycin treatment attenuated phosphorylation of P70S6K $\mathrm{K}^{\mathrm{Th} 389}$ and 

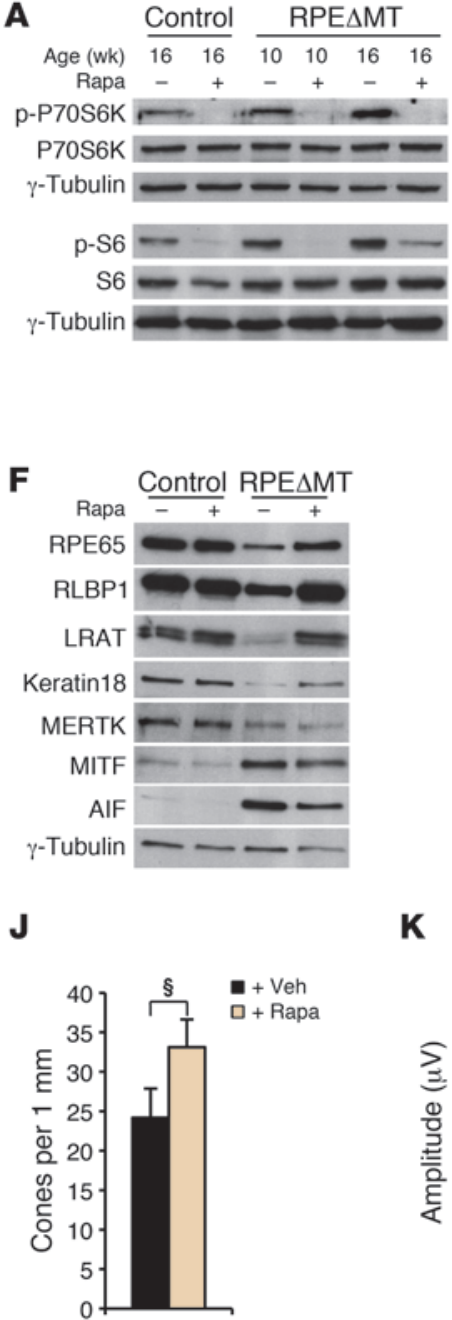

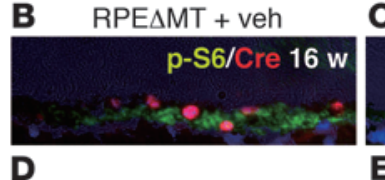

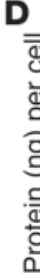
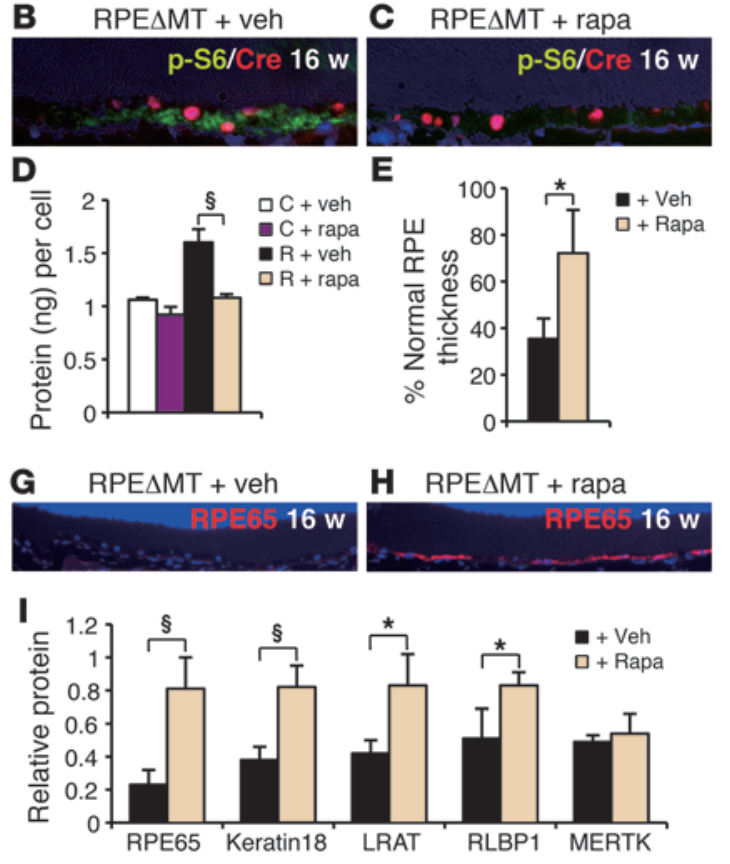

E

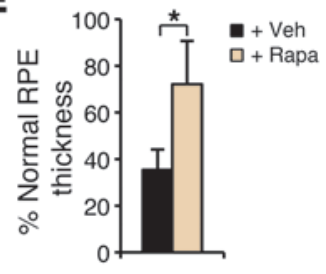

.
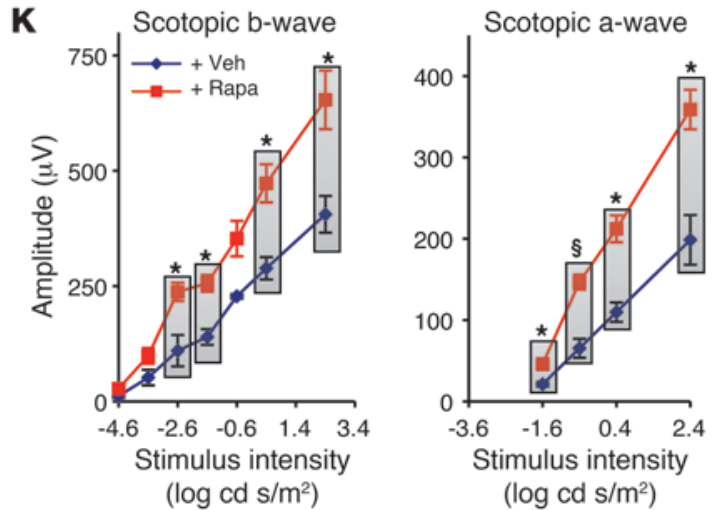

$\left(\log c d s / m^{2}\right)$

\section{Figure 7}

Rapamycin treatment attenuates loss of RPE-characteristic markers, RPE cell growth, and photoreceptor dysfunction in RPE $\triangle \mathrm{MT}$ mice. (A) An immunoblot shows reduced phosphorylation of

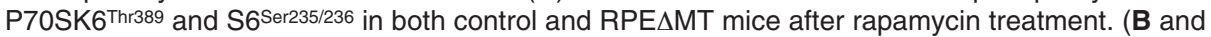
C) Immunostaining for $\mathrm{p}$-S6 $6^{\mathrm{Ser} 235 / 236}$ (green) and cre (red) shows reduced reactivity of p-S6 Ser235/236 $^{2}$ in cre-expressing RPE cells in rapamycin-treated (C) versus vehicle-treated (B) RPE $\triangle M T$ mice. Rapa, rapamycin. Original magnification, $\times 400$. (D) Rapamycin treatment normalizes cellular protein content in RPE $\Delta M T$ mice $(R+$ rapa) at 16 weeks of age (triplicates). (E) The percentage of RPE with normal thickness $(2.5-5 \mu \mathrm{m})$ is significantly increased in rapamycin-treated RPE $\Delta \mathrm{MT}$ mice at 22 weeks $(n=5)$, compared with that in vehicle-treated RPE $\Delta$ MT mice $(n=3)$. (F) An immunoblot demonstrates the normalizing effect of rapamycin on the levels of RPE characteristic proteins and AIF in RPE cells from 16-week-old RPE $\Delta M T$ mice. ( $G$ and $\mathbf{H}$ ) RPE65 reactivity (red) is preserved in RPE $\Delta$ MT mice by rapamycin treatment $(\mathbf{H})$. Original magnification, $\times 200$. (I) Quantification of proteins (triplicates) detected by immunoblot (e.g., F) shows rapamycin-induced increases in several RPE-characteristic markers in RPE $\triangle M T$ RPE cells (normalized to vehicle-treated control mice). (J) Rapamycin treatment of RPE $\Delta \mathrm{MT}$ mice $(n=6)$ preserves cone density at 22 weeks, compared with that of vehicle-treated RPE $\Delta$ MT mice $(n=3)$. (K) Electroretinography demonstrates significantly increased scotopic responses in 22-week-old RPE $\Delta$ MT mice treated with rapamycin $(n=5)$, compared with those of vehicle-treated RPE $\Delta$ MT mice $(n=4)$. Verticle bars clarify the 2 groups of values used for statistical comparison. Data represent mean \pm SEM. ${ }^{\star} P<0.05 ; \S P<0.01$.

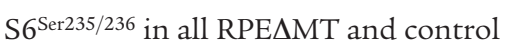
mice tested at 10 weeks of age or older (Figure 7A), reduced S6 6 Ser235/236 phosphorylation specifically in cre-expressing cells (Figure 7C), and normalized RPEAMT RPE cellular protein content (Figure 7D). While rapamycin did not alter RPE morphology in controls (Supplemental Figure 9, C and D), rapamycin-mediated attenuation of S6 phosphorylation in RPE $\Delta \mathrm{MT}$ creexpressing cells correlated with a preserved RPE phenotype at 15 weeks of age with respect to melanosome distribution, nuclear alignment, and uniformity of RPE thickness (Supplemental Figure 9, A and B). At 22 weeks, when normal RPE cells were not seen in vehicle-treated RPE $\triangle M T$ mice (Supplemental Figure 9E), rapamycin-treated RPEAMT mice had both normal and abnormal appearing RPE cells (Supplemental Figure 9, F and G). Specifically, the percentage of RPE across the retina with normal thickness $(2.5-5 \mu \mathrm{m})$ was significantly increased by rapamycin in RPE $\triangle M T$ mice (Figure 7E).

Rapamycin treatment of RPE $\Delta M T$ mice also preserved the expression levels at 16 weeks of several RPE-characteristic proteins, including RPE65, RLBP1, LRAT, and keratin 18 (Figure 7, F and I), and increased RPE65 reactivity was present in retinal sections of all rapamycin-treated RPE $\Delta \mathrm{MT}$ mice tested (e.g., Figure 7H). Interestingly, the level of AIF protein in RPEAMT mice decreased in response to rapamycin, as did the RPE cell fate determinant MITF (Figure 7F). Consistent with the preserved RPE differentiated phenotype and protein expression, rapamycin-treated RPE $\Delta M T$ mice have increased numbers of cones and an improved retinal scotopic ERG response at 22 weeks of age (Figure 7 , $\mathrm{J}$ and $\mathrm{K}$ ). The beneficial effects of rapamycin treatment did not extend to maintenance of RPE integrity and inhibition of EMT-like changes in RPE $\triangle M T$ mice at 24 weeks of age (Supplemental Figure 9, H-W). These changes probably result from activation of the HGF/c-Met pathway (65), and neither HGF nor phosphorylated c-Met levels in RPE $\triangle M T$ RPE were altered by rapamycin treatment (data not shown). Thus, inhibition of mTORC1 counteracts several of the initial pathological consequences of 

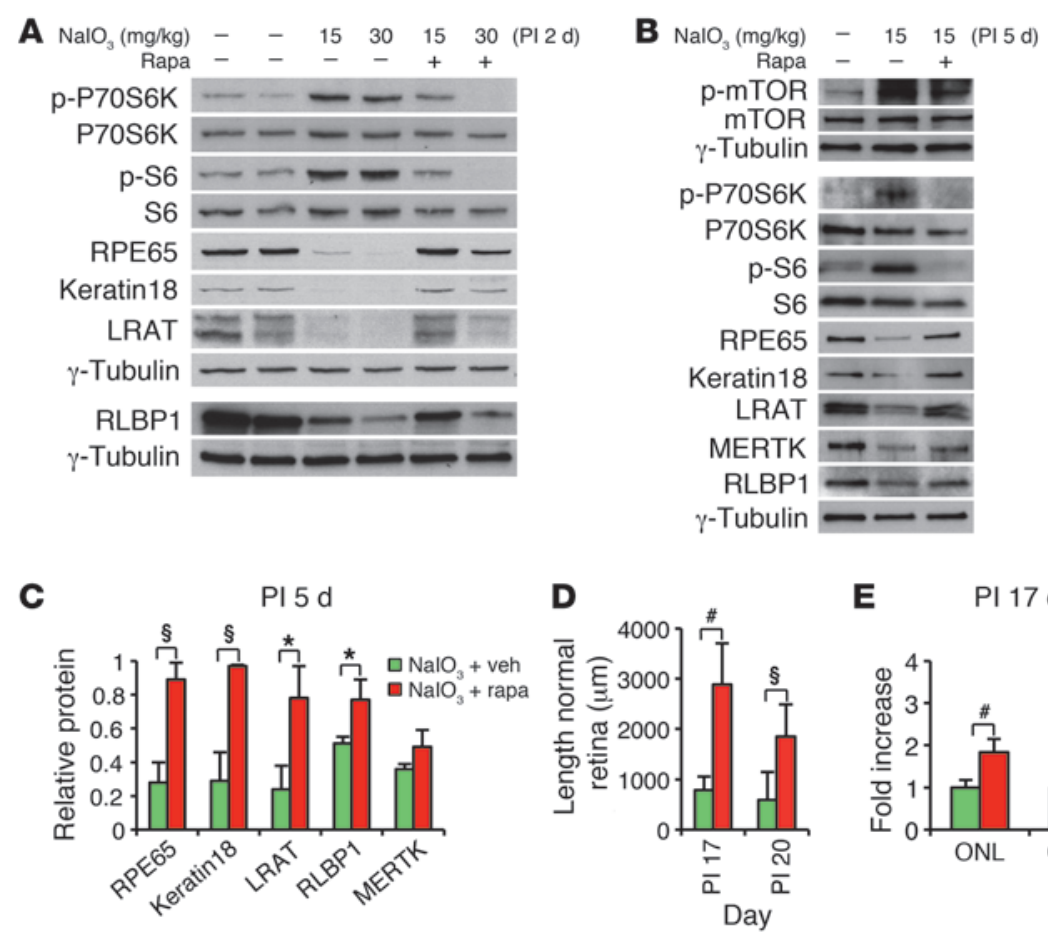

E $\quad$ PI $17 d$

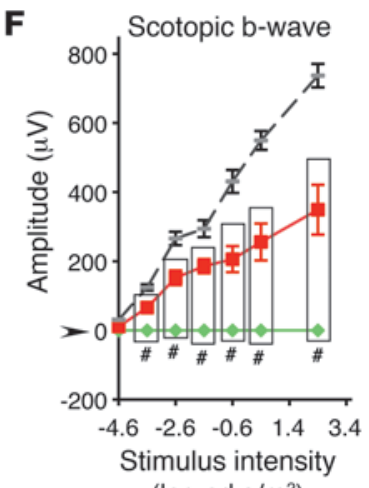

$\left(\log c d s / m^{2}\right)$

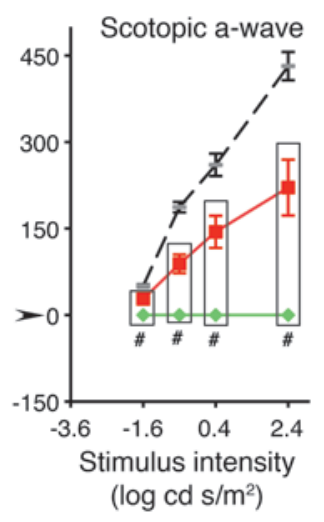

\section{Figure 8}

Rapamycin inhibits mTOR/S6K-mediated RPE dedifferentiation and subsequent retinal degeneration induced by $\mathrm{NaIO}_{3}$ in $\mathrm{B} 6$ mice. (A) Immunoblot of proteins from eyecups shows elevated phosphorylation of P70SK6 ${ }^{\text {Thr } 389}$ and S6 $6^{\text {Ser235/236 }}$ and loss of RPE markers 2 days $\mathrm{PI}$ of $\mathrm{NaIO}_{3}$ (2 middle lanes). (B) At day $5 \mathrm{PI}$, an immunoblot detects persistent activation of P70SK6 ${ }^{\text {Thr } 389}$ and S6 Ser235/236 and diminished RPE markers in isolated RPE cells (middle lane). The effects are inhibited by rapamycin treatment (A, 2 right lanes; $\mathbf{B}$, right lane). (C) Quantification of RPE proteins 5 days $\mathrm{PI}$ of $\mathrm{NaIO}_{3}$ (triplicates) shows preservation of several RPE markers after rapamycin treatment (red bars) (normalized to PBS and vehicle-treated controls). (D and E) Quantification of the length of normal retina (RPE and neural retina) at PI days 17 and 20 (D), and outer nuclear layer thickness and cone density at PI day 17 (E). Rapamycin treatment (red bars, $n=6$ ) results in a striking preservation of retinal morphology relative to that of vehicle treatment (green bars, $n=6$ ) in $\mathrm{NaIO}_{3}$-injected mice. In $\mathbf{E}$, the mean value of outer nuclear layer and cones of vehicletreated mice was defined as 1 . Data represent the relative fold increase of the corresponding value obtained from rapamycin-treated, $\mathrm{NaIO}_{3}$-injected mice. (F) Electroretinography demonstrates significantly increased scotopic and photopic responses at $\mathrm{PI}$ day 17 in $\mathrm{NalO}_{3}$ injected $\mathrm{B} 6$ mice treated with rapamycin (red, $n=5$ ), compared with those in vehicle-treated $\mathrm{NaIO}_{3}$-injected controls (green, $n=6$ ). The dashed curve represents control mice (PBS + vehicle, $n=7$ ). Verticle bars clarify the 2 groups of values used for statistical comparison. Data represent mean \pm SEM. ${ }^{*} P<0.05$; $\S P<0.01 ; \# P<0.001$.
OXPHOS deficiency in the RPE as well as subsequent photoreceptor deficits, at least up to 22 weeks of age.

$m T O R / S 6 K / S 6$ pathway activation causes RPE dedifferentiation and photoreceptor degeneration in response to oxidative stress. Sodium iodate $\left(\mathrm{NaIO}_{3}\right)$ is a toxin known to induce selective RPE degeneration, presumably by oxidative stress, and secondary retinal degeneration $(34,66)$. While C57BL/6 (B6) mice treated with $50 \mathrm{mg} / \mathrm{kg}$ $\mathrm{NaIO}_{3}$ experience massive RPE cell loss within 7 days, a lower dose (30-35 mg/kg) does not cause swift RPE degeneration (66) but does induce rapid activation of AKT and reduction of RPE65 (34). Moreover, $\mathrm{Hg} f \mathrm{mRNA}$ expression in the RPE increases acutely after $\mathrm{NaIO}_{3}$ injection (67). Although we found no evidence of increased oxidative damage to nucleic acids in RPE $\triangle \mathrm{MT}$ mice as old as 8 months (data not shown), the similarities in AKT activation, $\mathrm{Hg}$ induction, and RPE65 loss suggested that upregulation of the mTOR/S6K/S6 pathway might also play an important role in the $\mathrm{RPE}$ response to oxidative stress.

We injected $\mathrm{B} 6$ mice with $\mathrm{NaIO}_{3}$ at 2 doses, $15 \mathrm{mg} / \mathrm{kg}$ and $30 \mathrm{mg} /$ $\mathrm{kg}$, or with PBS for controls. $\mathrm{NaIO}_{3}$-injected mice were subgrouped for daily rapamycin or vehicle treatment. We found that $\mathrm{NaIO}_{3}$ does indeed result in RPE oxidative damage (Supplemental Figure 10, B-D), which we believe has not been shown previously for any dose. Activities of 2 key OXPHOS enzymes (COX/SDH) were not diminished in the RPE 5 days PI (Supplemental Figure 10, F and G), indicating that $\mathrm{NaIO}_{3}$ treatment is an oxidative and qualitatively different stress than OXPHOS deficiency. Despite this difference, $\mathrm{NaIO}_{3}$, like OXPHOS deficiency, induced phosphorylation of P70SK6 ${ }^{\text {Thr389 }}$

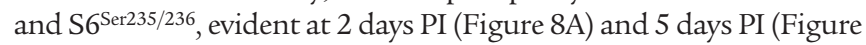
$8 \mathrm{~B}$ ), and treatment with rapamycin blunted these changes (Figure 8, $\mathrm{A}$ and $\mathrm{B}$ ). Both doses of $\mathrm{NaIO}_{3}$ induced a rapid reduction in the levels of RPE65 and several other RPE-characteristic proteins (Figure 8A), similar to the early changes in RPEAMT mice (Figure 2L). As it did for OXPHOS deficiency (Figure 7F), rapamycin counteracted loss of these markers (Figure 8, B and C). There are also parallels between the 2 stresses in longer-term consequences. At 17 days $\mathrm{PI}, \mathrm{NaIO}_{3}(15$ $\mathrm{mg} / \mathrm{kg}$ ) caused widespread RPE histological abnormalities and photoreceptor degeneration (Supplemental Figure 10, I-K), with RPE cell thickening and multilayers (Supplemental Figure 10J) or, more often, rounded cells protruding into the subretinal space (Supplemental Figure 10I), features similar to those seen in older RPE $\triangle M T$ 
mice (e.g., 52 weeks, Supplemental Figure 5A). Rapamycin significantly increased the length of histologically normal RPE and neural retina (Figure 8D and Supplemental Figure 10, K-M) as well as the number of all photoreceptors (Figure 8E and Supplemental Figure $10 \mathrm{~K}$ ) and, particularly, the number of cones (Figure 8E). Consistent with our histological assessment, $\mathrm{NaIO}_{3}$-treated mice $(15 \mathrm{mg} / \mathrm{kg})$ had severely diminished ERG responses at 17 days PI, and this functional impairment was also significantly ameliorated by rapamycin (Figure 8F). Thus, mTOR activation plays a crucial role in the initial response of the RPE to both metabolic and oxidative stresses. Inhibition of this stress response has beneficial consequences for the RPE and photoreceptors, highlighting $\mathrm{mTOR}$ as a potential therapeutic target for retinal degenerations that primarily involve RPE stress.

\section{Discussion}

We have identified a common response of the adult murine RPE to diverse stressors. The stress response arises from activation of the $\mathrm{PI} 3 / \mathrm{AKT} / \mathrm{mTOR}$ signaling pathway. A crucial consequence of the response is enhanced RPE cell survival. We found scant evidence of RPE cell death under conditions of chronic OXPHOS deficiency or acute chemical oxidative damage. Activation of AKT is a well-known mechanism for engaging cell survival pathways $(59,62)$. AKT-mediated phosphorylation of BAD, which we detected in RPE $\triangle M T R P E$, directly inhibits apoptosis (59). In the case of chronic OXPHOS deficiency, AKT is presumably activated via the HGF/c-Met pathway, which we demonstrated is essential for RPE cell viability. For acute chemical oxidative stress, AKT activation may occur by direct oxidation of PTEN (34), a negative regulator of PI3K activity. While we found no evidence supporting a role for PTEN in AKT activation in RPE $\triangle \mathrm{MT}$ mice, probably due to a lack of oxidative stress, we and others (67) do detect HGF induction in $\mathrm{NaIO}_{3}$-treated mice. Regardless of the mechanism of AKT activation, our results highlight the durable nature of RPE cells in vivo and suggest an alternative to a prevailing tenet of AMD pathogenesis that emphasizes the role of RPE apoptosis in disease etiology $(10,13)$.

RPE dedifferentiation and hypertrophy are also cardinal features of the stress response. The AKT/mTOR axis regulates biosynthetic programs during cell proliferation $(61,62,68)$. AKT-mediated activation of mTOR leads to a general increase in protein and lipid synthesis through S6K-mediated phosphorylation of ribosomal protein S6 and SREBP-mediated stimulation of lipogenesis (63), respectively. The RPE stress response entails minimal cell division, and the result is an increase in cellular biomass and a thickened RPE. In contrast to the increase in total cellular protein, mTOR activation paradoxically results in diminished expression of a number of RPE-characteristic proteins, including several necessary for specialized RPE functions such as enzymatic conversion of all-trans-retinal to 11-cis-retinal (RPE65, LRAT, and RLBP1) and phagocytosis of photoreceptor outer segment tips (MERTK). The rapidity of the loss of these proteins after $\mathrm{NaIO}_{3}$ treatment is consistent with an effect on protein stability or translation, but for OXPHOS deficiency, we also found diminished steady-state transcript levels (Supplemental Figure 3Q), suggesting a partial reprogramming of the RPE cell from a fully differentiated, quiescent epithelial phenotype, to a less differentiated growth mode. mTOR-mediated tissue dedifferentiation has also been described for vascular (69) and bladder (70) smooth muscle. RPE $\triangle M T$ RPE eventually exhibits a number of features characteristic of an EMT, a likely cause of which is activation of the HGF/c-Met pathway (65). Thus, RPE cell survival in the context of the stress response comes at the expense of epithelial attributes.
RPE cell survival also comes at a cost to photoreceptors. In both stress models, RPE dedifferentiation and hypertrophy correlated with death of adjacent photoreceptors. Given the manifold functions performed by the RPE, the factors contributing to neuronal cell death are likely complex. In RPE $\triangle \mathrm{MT}$ mice, RPE hypertrophy, loss of cell polarization, and increased RPE glycolytic flux probably result in diminished delivery to the outer neural retina of nutrients in general and glucose in particular (Supplemental Figure 6F). Glucose deprivation has been implicated as a cause of cone degeneration in murine models of RP arising from mutation of rod-specific genes (71). A clue to another possible cause of photoreceptor degeneration in RPE $\triangle \mathrm{MT}$ mice is the regional loss of cones and relative preservation of rods. Whereas both rod and cone opsins require 11-cis-retinal to initiate phototransduction, lack of the chromophore in mice leads to a rapid degeneration of central and ventral cones due to the failure of opsins and other membrane-associated cone signaling proteins to traffic appropriately to outer segments (72). One of the earliest consequences of RPE dedifferentiation in both stress models is a striking reduction in the amount of RPE65, a protein required for the production in the RPE of 11-cis-retinal. This, along with reductions in other visual cycle proteins, such as LRAT and RLBP1, should cause a significant decline in the level of the chromophore in areas affected by the stress response, resulting in abnormal cone morphogenesis. In addition to regional cone deficits, low 11-cis-retinal levels may contribute to the diminished scotopic and photopic ERG responses in both models. Moreover, both RPEAMT mice and B6 (73) $\mathrm{NaIO}_{3}$-treated mice express only the RPE65-MET450 allele, the steady-state level of which is substantially lower than that of the LEU450 variant (73), which may accentuate the 11-cis-retinal deficit and exacerbate the deleterious effects of RPE dedifferentiation on photoreceptor morphology and function. Our findings suggest that stress-induced failure of RPE cell functions, well in advance of overt RPE cell loss, may contribute to the pathogenesis of human retinal degeneration.

A variety of perturbations seem to trigger the stress response in the adult murine RPE in vivo. Injection of oligomeric amyloid $\beta$ peptide into the subretinal space of B6 mice causes RPE hypertrophy and hypopigmentation (but not apoptosis) and diminished expression of RLBP1, RPE65, and tight junction proteins (74). Mice homozygous for a null mutation in the hemochromatosisassociated gene, Hfe, exhibit late-onset (18 months) RPE hypertrophy and hyperplasia, presumably as a result of iron accumulation (75). Similarly, iron buildup in the RPE of ceruloplasmin/hephaestin double-knockout mice leads to RPE hyperplasia and hypertrophy, with increased autofluorescent deposits in 7- to 9-month-old animals (76). In vivo ribozyme-mediated knockdown of murine manganese superoxide dismutase, which is encoded by the nuclear gene Sod 2 and which localizes to the mitochondrial matrix, causes "distended" RPE with vacuoles and autofluorescent aggregates (77). A common theme among these models is increased oxidative damage in the RPE, which may explain similarities to RPE pathology in $\mathrm{NaIO}_{3}$-injected mice. However, similarities to RPEAMT mice, in which the RPE undergoes metabolic rather than oxidative stress, indicate the response is more general.

Evidence suggests that the AKT/mTOR stress response is relevant to human retinal degenerative diseases. RPEAMT mice exhibit a number of features evocative of those seen in human disorders. These include RPE hypertrophy with vacuoles; loss of cell polarization, adhesion, and normal hexagonal morphology; abnormal 
proliferation and apical migration, leading to the appearance of hyperplasia; increased autofluorescence consistent with A2E-containing lipofuscin; pigmentation abnormalities; attenuation and atrophy; and diminished photoreceptor function with subsequent degeneration. A clinicopathological study of adult vitelliform macular degeneration lesions in eyes also affected with AMD describes a sequence of changes in the macular RPE as "first undergoing hypertrophy and then disruption and attenuation" (78). While mice do not have maculae, this description is remarkably similar to the progression we have documented in RPE $\triangle \mathrm{MT}$ mice (Figure $3 \mathrm{R}$ ). In some cases of adult vitelliform macular degeneration, RPE microvilli were absent and foveal cones were lost or "reduced to stumps" (78), suggesting a process of RPE dedifferentiation and cone dysmorphogenesis. An analysis of postmortem eyes from an individual with retinopathy due to Kearns-Sayre syndrome, which is caused by mtDNA mutations, reported a marked decrease in the number of apical microvilli and basal infoldings, along with a predominance of irregular polygonal RPE cells and hypopigmentation with disruption of the normal polarity of apical melanin (23). RPE hypertrophy has also been described in advanced AMD (48) and may be more common at earlier stages of the disease or in particular cellular environments. For example, the proangiogenic effect of interferon $\gamma$ on VEGF secretion by human RPE cells in culture is mediated by mTOR (79), and RPE hypertrophy and hyperplasia have been described near disciform scars in AMD eyes (80). Thus, the AKT $/ \mathrm{mTOR}$ stress response is likely a characteristic of both the adult murine and human RPE.

The positive effects of mTORC1 inhibition on the RPE and photoreceptors, for both chronic metabolic stress and acute oxidative stress, are quite remarkable. Daily rapamycin treatment of RPE $\Delta M T$ mice, beginning at 4 weeks, normalized RPE protein expression 3 months later in terms of both total cellular protein and specific proteins; markers of mature RPE increased in abundance, whereas MITF and proteins upregulated in response to OXPHOS deficiency (e.g., AIF and COX4) decreased in abundance, approaching levels in cre-negative controls. Rapamycin-induced protein normalization indicates a retardation of RPE dedifferentiation, which manifested as reduced RPE hypertrophy with improved morphological appearance, preservation of cones, and enhanced scotopic and photopic ERG responses. Similarly, rapamycin treatment of $\mathrm{NaIO}_{3}$-injected mice normalized protein expression, preserved photoreceptor numbers, augmented scotopic and photopic ERG responses at 17 days PI, and more than tripled the length of histologically normal retina at 17 and 20 days PI. Rapamycin is approved in the United States for renal transplantation and as a treatment for renal cancer and is currently being evaluated in a clinical trial (NCT00766649) for the treatment of the geographic atrophy form of dry AMD, the stated rationale for which is the antiinflammatory properties of the drug. While it is possible that the antiinflammatory effects of rapamycin contribute to the beneficial responses we observed, we believe that any such contribution is likely to be, at most, small. We saw no obvious increase in retinal macrophages/microglia in RPE $\triangle M T$ mice during RPE dedifferentiation, the stage at which rapamycin was administered (e.g., Supplemental Figure 4N). Moreover, the RPE was the only site of increased mTOR signaling that we detected. Our results thus provide an alternative rationale for the trial of rapamycin in geographic atrophy and a similar trial for the treatment of neovascular AMD (NCT00712491). Inhibition of $\mathrm{mTOR}$ is a plausible therapeutic strategy for human retinal degenerative diseases that involve RPE stress.
Although rapamycin significantly slows dedifferentiation and growth while maintaining RPE viability, the drug does not completely reverse the negative effects of RPE metabolic or oxidative stress. This may result from processes not affected by mTORC1 inhibition. In fact, rapamycin treatment can actually enhance activation of AKT through loss of feedback inhibition (61) as well as cause activation of the MAP kinase, ERK (81). A multimodal approach, analogous to the type pursued in oncology, in which several key pathways are inhibited simultaneously (82), may prove more effective. However, as the consequences of c-Met inhibition starkly illustrate, activation of critical cell survival pathways must remain intact.

In summary, we have characterized a general in vivo stress response of the RPE, in which cells prolong their survival but lose important specialized and general epithelial attributes, with devastating consequences for neighboring photoreceptors. Recognition of the response provides insight into human retinal degenerative diseases involving RPE stress, while the beneficial effects of early inhibition of the mTORC1 arm of the response suggest a new rationale for therapy of these disorders.

\section{Methods}

Animals. BEST1-cre transgenic mice, in which cre recombinase is controlled by a fragment of the human BEST1 promoter $(-585$ to +38$)$, were generated on a B6 background. Pigmented and albino mice with a loxP-flanked Tfam allele (Tfam ${ }^{\text {loxp/loxp }}$ ) on a mixed B6/129 background were provided by Nils-Göran Larsson (Karolinska Institute, Stockholm, Sweden) and have been described previously $(36,83)$. Both pigmented and albino mice with

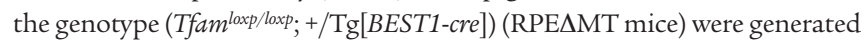
by breeding female BEST1-cre transgenic mice with pigmented and albino

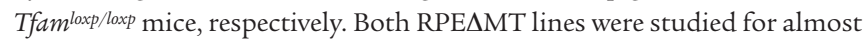
all experiments, and cre-negative littermates served as controls, unless otherwise stated. B6 mice were purchased from The Jackson Laboratory and Charles River Laboratories. All the mice in this study were homozygous for the Rpe65 $5^{\mathrm{M} 50}$ allele. All procedures were in compliance with the Association for Research in Vision and Ophthalmology Statement for the use of Animals in Ophthalmic and Visual Research and were approved by Stanford University Administrative Panel on Laboratory Animal Care.

Reagents, antibodies, and oligonucleotides. All reagents were purchased from Sigma-Aldrich, unless otherwise stated. Antibodies and oligonucleotides are detailed in Supplemental Tables 1 and 2.

Tissue preparation. For preparation of eyecups, eyes were enucleated and rinsed with 1X PBS (Mediatech). After trimming connective tissues, the anterior portion was removed, and the neural retina was carefully separated from the posterior eyecup. RPE cells were isolated as previously described $(34,84)$. Briefly, eyecups (with neural retina) were treated with hyaluronidase $(3.2 \mathrm{U} / \mathrm{ml})$ and collagenase $(195 \mu \mathrm{g} / \mathrm{ml})$, or dispase $(200 \mathrm{U} / \mathrm{ml})$, in $1 \mathrm{X}$ HBSS at $37^{\circ} \mathrm{C}$ for 30 minutes. Following an HBSS wash, the neural retina was removed, and RPE cells were carefully isolated in 1X HBSS containing protease inhibitors. For cell counting, cells were further dissociated with $0.25 \%$ trypsin-EDTA for 5 minutes and counted with a hemocytometer.

Quantitative RT-PCR and in situ bybridization. RNA extraction and RT-PCR were done as described previously (85). Quantitative PCR (Q-PCR) was performed with addition of SYBR Green I (Bio-Rad) intercalating dye, and data were collected using the iCycler (Bio-Rad). Relative expression analysis was performed as described previously (86). In situ hybridization with digoxygenin-labeled probes for Cox 1 and Rpe65 was carried out on cryosections as described previously (87). Oligonucleotide primers for Q-PCR and probe production are detailed in Supplemental Table 1 and Supplemental Methods.

Immunofluorescence microscopy. Cryosections and RPE flat mounts were prepared as described previously $(88,89)$ and then blocked with $5 \%$ normal 
goat serum/0.1\% Triton X-100/1X PBS at room temperature for 1 hour. Primary antibodies were incubated overnight at $4{ }^{\circ} \mathrm{C}$ followed by appropriate secondary antibodies (Supplemental Table 2). Slides were mounted with mounting medium containing DAPI (Vector Laboratories). RPE flat mounts were counterstained by Hoechst 33342 and mounted by regular Vectashield mounting medium (Vector Laboratories). FITC-palloidin and FITC-PNA staining (both from Invitrogen) were used in an antibody staining procedure, according to the manufacturer's instructions. General fluorescent microscopy was performed as described previously (88). Confocal microscopy was carried out on Lecia SP2 microscope (Leica Microsystems). Images from Z-series were taken in a Z-projection and merged by Volocity imaging software (PerkinElmer), which also generated Z-stack images.

Animal treatments. For BrdU labeling, RPE $\triangle \mathrm{MT}$ mice were selected $(n=3$ for each group) with equal numbers of littermate controls. BrdU (EMD Inc.) was diluted to $5 \mathrm{mg} / \mathrm{ml}$ in $1 X$ PBS. Mice received intraperitoneal injections of $\mathrm{BrdU}(50 \mathrm{mg} / \mathrm{kg}$ ) for 6 consecutive days. Albino RPE$\Delta \mathrm{MT}$ mice were selected for treatment with PHA-665752 (Tocris Bioscience), a selective c-Met inhibitor, or vehicle ( $n=6$ for each group). Littermate controls were also administrated with PHA-665752 $(n=3)$ or vehicle $(n=3)$. Preparation and injection of active PHA-665752 $(3.3 \mathrm{mg} / \mathrm{ml}$ and approximately $150 \mu \mathrm{l} / \mathrm{mouse} / \mathrm{d})$ were done as previously described (55). Mice were injected with active PHA-665752 $(20 \mathrm{mg} / \mathrm{kg})$ or vehicle (10\% polyethyleneglycol 400 and L-lactate at $\mathrm{pH} 3.5)$ via tail vein for 6 consecutive days. For rapamycin treatment, 4-week-old pigmented RPE $\triangle \mathrm{MT}$ mice were intraperitoneally injected daily with rapamycin $(3 \mathrm{mg} / \mathrm{kg})(n=15)$ or vehicle (4\% ethanol, $5 \%$ Tween 80 , and $5 \%$ polyethyleneglycol 400) $(n=15)$ until sacrifice. Littermate controls also received rapamycin $(n=14)$ or vehicle $(n=20)$. Rapamycin was prepared as described elsewhere (90). $\mathrm{For} \mathrm{NaIO}_{3}$ and rapamycin studies, $\mathrm{B} 6$ mice were intravenously injected once via tail vein with $1 \mathrm{X} \mathrm{PBS}$ or $\mathrm{NaIO}_{3}$ (diluted to $5 \mathrm{mg} / \mathrm{ml}$ in $1 \mathrm{X}$ PBS). Rapamycin or vehicle treatment was done as described above, beginning 12 hours prior to $\mathrm{NaIO}_{3}$ injection, because the effect of intravenous $\mathrm{NaIO}_{3}$ is rapid (34), whereas the effect of intraperitoneally injected rapamycin only accumulates over time. Mice were grouped for biochemical studies as PBS with vehicle (group A, $n=6$ ), $\mathrm{NaIO}_{3}(15 \mathrm{mg} / \mathrm{kg}, \sim 75 \mu \mathrm{l} /$ mouse) with vehicle (group $\mathrm{B}, n=6), \mathrm{NaIO}_{3}\left(15 \mathrm{mg} / \mathrm{kg}\right.$ ) with rapamycin (group C, $n=6$ ), $\mathrm{NaIO}_{3}$ (30 mg/kg, 150 $\mu \mathrm{l} /$ mouse) with vehicle (group D, $n=4)$, and $\mathrm{NaIO}_{3}(30 \mathrm{mg} /$ $\mathrm{kg}$ ) with rapamycin (group E, $n=4$ ). Animals for ERG and histological studies were assigned as PBS with vehicle (group a, $n=8)$ and $\mathrm{NaIO}_{3}(15 \mathrm{mg} / \mathrm{kg}$ ) with vehicle (group b, $n=12$ ) or with rapamycin (group c, $n=12$ ).

Statistics. An unpaired 2-tailed Student's $t$ test was performed. $P<0.05$ was considered significant. Data in figures represent mean \pm SD unless otherwise stated. The average deviation is equal to the sum of the absolute values of the individual deviations divided by the number of deviations and represents the average of the absolute deviations of data points from their mean.

\section{Acknowledgments}

We thank members of the Vollrath Laboratory for discussions; David Strick for comments on the manuscript; Dritan Agalliu, Paul Buckmaster, and Jacque Duncan for advice; and Hannes Vogel for help with histochemical staining. This work was supported by grants from the NIH (EY014650; EY001919; EY00331; EY015240; P30EY002162), Foundation Fighting Blindness, Thome Memorial Foundation, and Research to Prevent Blindness and by a Stanford Medical School Dean's Postdoctoral Fellowship.

Received for publication July 9, 2010, and accepted in revised form October 22, 2010.

Address correspondence to: Douglas Vollrath, Department of Genetics, 300 Pasteur Drive, Lane Building L305, Stanford University School of Medicine, Stanford, California 94305-5120, USA. Phone: 650.723.3290; Fax: 650.723.7016; E-mail: vollrath@stanford.edu.

Chen Zhao's present address is: Tianjin Key Laboratory of Ophthalmology and Vision Science, Tianjin Eye Hospital, Tianjin Medical University, Tianjin, China.
1. Foulds WS. The choroidal circulation and retinal metabolism--an overview. Eye (Lond). 1990; 4(pt 2):243-248.

2. Strauss $O$. The retinal pigment epithelium in visual function. Physiol Rev. 2005;85(3):845-881.

3. Thompson DA, Gal A. Vitamin A metabolism in the retinal pigment epithelium: genes, mutations, and diseases. Prog Retin Eye Res. 2003;22(5):683-703.

4. Gal A, et al. Mutations in MERTK, the human orthologue of the RCS rat retinal dystrophy gene, cause retinitis pigmentosa. Nat Genet. 2000;26(3):270-271.

5. Strick DJ, Vollrath D. Focus on molecules: MERTK [published online ahead of print May 22, 2010]. Exp Eye Res. doi:10.1016/j.exer.2010.05.006.

6. Petrukhin $\mathrm{K}$, et al. Identification of the gene responsible for Best macular dystrophy. Nat Genet. 1998;19(3):241-247.

7. Davidson AE, et al. Missense mutations in a retinal pigment epithelium protein, bestrophin-1, cause retinitis pigmentosa. Am J Hum Genet. 2009; 85(5):581-592.

8. Weber BH, Vogt G, Pruett RC, Stohr H, Felbor U. Mutations in the tissue inhibitor of metalloproteinases-3 (TIMP3) in patients with Sorsby's fundus dystrophy. Nat Genet. 1994;8(4):352-356.

9. Stone EM, et al. A single EFEMP1 mutation associated with both Malattia Leventinese and Doyne honeycomb retinal dystrophy. Nat Genet. 1999; 22(2):199-202.

10. Dunaief JL, Dentchev T, Ying GS, Milam AH. The role of apoptosis in age-related macular degeneration. Arch Ophthalmol. 2002;120(11):1435-1442.

11. Zarbin MA. Current concepts in the pathogenesis of age-related macular degeneration. Arch Ophthal- mol. 2004;122(4):598-614.

12. Jager RD, Mieler WF, Miller JW. Age-related macular degeneration. NEngl J Med. 2008;358(24):2606-2617.

13. Liang FQ, Godley BF. Oxidative stress-induced mitochondrial DNA damage in human retinal pigment epithelial cells: a possible mechanism for RPE aging and age-related macular degeneration. Exp Eye Res. 2003;76(4):397-403.

14. Jarrett SG, Lin H, Godley BF, Boulton ME. Mitochondrial DNA damage and its potential role in retinal degeneration. Prog Retin Eye Res. 2008; 27(6):596-607.

15. Hageman GS, Luthert PJ, Victor Chong NH, Johnson LV, Anderson DH, Mullins RF. An integrated hypothesis that considers drusen as biomarkers of immunemediated processes at the RPE-Bruch's membrane interface in aging and age-related macular degeneration. Prog Retin Eye Res. 2001;20(6):705-732.

16. Yu DY, Cringle SJ. Retinal degeneration and local oxygen metabolism. Exp Eye Res. 2005;80(6):745-751.

17. Suter M, et al. Age-related macular degeneration. The lipofusion component $\mathrm{N}$-retinyl-N-retinylidene ethanolamine detaches proapoptotic proteins from mitochondria and induces apoptosis in mammalian retinal pigment epithelial cells. J Biol Chem. 2000;275(50):39625-39630.

18. Vives-Bauza C, et al. The age lipid A2E and mitochondrial dysfunction synergistically impair phagocytosis by retinal pigment epithelial cells. J Biol Chem. 2008;283(36):24770-24780.

19. Karunadharma PP, Nordgaard CL, Olsen TW, Ferrington DA. Mitochondrial DNA damage as a potential mechanism for age-related macular degeneration. Invest Ophthalmol Vis Sci. 2010;51(11):5470-5479.
20. Canter JA, et al. Mitochondrial DNA polymorphism A4917G is independently associated with age-related macular degeneration. PLoS One. 2008; 3(5):e2091.

21. SanGiovanni JP, et al. Mitochondrial DNA variants of respiratory complex I that uniquely characterize haplogroup T2 are associated with increased risk of age-related macular degeneration. PLoS One. 2009;4(5):e5508.

22. Kanda A, et al. A variant of mitochondrial protein LOC387715/ARMS2, not HTRA1, is strongly associated with age-related macular degeneration. Proc Natl Acad Sci U S A. 2007;104(41):16227-16232.

23. Eagle RC Jr, Hedges TR, Yanoff M. The KearnsSayre syndrome: a light and electron microscopic study. Trans Am Ophthalmol Soc. 1982;80:218-234.

24. Massin P, et al. Macular pattern dystrophy associated with a mutation of mitochondrial DNA. Am J Ophthalmol. 1995;120(2):247-248.

25. Rath PP, et al. Characterisation of the macular dystrophy in patients with the A3243G mitochondrial DNA point mutation with fundus autofluorescence. Br J Ophthalmol. 2008;92(5):623-629.

26. Zhang C, Baffi J, Cousins SW, Csaky KG. Oxidantinduced cell death in retinal pigment epithelium cells mediated through the release of apoptosis-inducing factor. J Cell Sci. 2003;116(pt 10):1915-1923.

27. Jin M, Yaung J, Kannan R, He S, Ryan SJ, Hinton DR. Hepatocyte growth factor protects RPE cells from apoptosis induced by glutathione depletion. Invest Ophthalmol Vis Sci. 2005;46(11):4311-4319.

28. Vander Heiden MG, Cantley LC, Thompson CB. Understanding the Warburg effect: the metabolic requirements of cell proliferation. Science. 2009; 
324(5930):1029-1033.

29. Miceli MV, Newsome DA, Schriver GW. Glucose uptake, hexose monophosphate shunt activity, and oxygen consumption in cultured human retinal pigment epithelial cells. Invest Ophthalmol Vis Sci. 1990;31(2):277-283.

30. Glocklin VC, Potts AM. The metabolism of retinal pigment cell epithelium. Ii. Respiration and glycolysis. Invest Ophthalmol. 1965;4:226-234.

31. Winkler BS, Sauer MW, Starnes CA. Modulation of the Pasteur effect in retinal cells: implications for understanding compensatory metabolic mechanisms. Exp Eye Res. 2003;76(6):715-723.

32. Burke JM. Epithelial phenotype and the RPE: is the answer blowing in the Wnt? Prog Retin Eye Res. 2008;27(6):579-595.

33. Ramkumar HL, Zhang J, Chan CC. Retinal ultrastructure of murine models of dry age-related macular degeneration (AMD). Prog Retin Eye Res. 2010; 29(3):169-190.

34. Kim JW, Kang KH, Burrola P, Mak TW, Lemke G. Retinal degeneration triggered by inactivation of PTEN in the retinal pigment epithelium. Genes Dev. 2008; 22(22):3147-3157

35. Rattner A, Toulabi L, Williams J, Yu H, Nathans J. The genomic response of the retinal pigment epithelium to light damage and retinal detachment. J Neurosci. 2008;28(39):9880-9889.

36. Larsson NG, et al. Mitochondrial transcription factor $A$ is necessary for mtDNA maintenance and embryogenesis in mice. Nat Genet. 1998;18(3):231-236.

37. Esumi N, Oshima Y, Li Y, Campochiaro PA, Zack DJ. Analysis of the VMD2 promoter and implication of E-box binding factors in its regulation. J Biol Chem. 2004;279(18):19064-19073

38. Lacovelli J, et al. Generation of cre transgenic mice with postnatal RPE-specific ocular expression. Invest Ophthalmol Vis Sci. In press.

39. Wang J, et al. Dilated cardiomyopathy and atrioventricular conduction blocks induced by heartspecific inactivation of mitochondrial DNA gene expression. Nat Genet. 1999;21(1):133-137.

40. Silva JP, et al. Impaired insulin secretion and betacell loss in tissue-specific knockout mice with mitochondrial diabetes. Nat Genet. 2000;26(3):336-340.

41. Sorensen L, et al. Late-onset corticohippocampal neurodepletion attributable to catastrophic failure of oxidative phosphorylation in MILON mice. J Neurosci. 2001;21(20):8082-8090.

42. Ekstrand MI, et al. Progressive parkinsonism in mice with respiratory-chain-deficient dopamine neurons. Proc Natl Acad Sci U S A. 2007;104(4):1325-1330.

43. Metodiev MD, et al. Methylation of $12 \mathrm{~S}$ rRNA is necessary for in vivo stability of the small subunit of the mammalian mitochondrial ribosome. Cell Metab. 2009;9(4):386-397.

44. Kujoth GC, et al. Mitochondrial DNA mutations, oxidative stress, and apoptosis in mammalian aging. Science. 2005;309(5733):481-484.

45. Wang J, Silva JP, Gustafsson CM, Rustin P, Larsson NG. Increased in vivo apoptosis in cells lacking mitochondrial DNA gene expression. Proc Natl Acad Sci US A. 2001;98(7):4038-4043.

46. Lipton SA, Bossy-Wetzel E. Dueling activities of AIF in cell death versus survival: DNA binding and redox activity. Cell. 2002;111(2):147-150.

47. Mishima K, Handa JT, Aotaki-Keen A, Lutty GA, Morse LS, Hjelmeland LM. Senescence-associated beta-galactosidase histochemistry for the primate eye. Invest Ophthalmol Vis Sci. 1999;40(7):1590-1593.

48. Coleman HR, Chan CC, Ferris FL 3rd, Chew EY. Age-related macular degeneration. Lancet. 2008; 372(9652):1835-1845.

49. Bharti K, Nguyen MT, Skuntz S, Bertuzzi S, Arnheiter $\mathrm{H}$. The other pigment cell: specification and development of the pigmented epithelium of the vertebrate eye. Pigment Cell Res. 2006;19(5):380-394.

50. Liu Y, Xin Y, Ye F, Wang W, Lu Q, Kaplan HJ, Dean
DC. Taz-Tead1 links cell-cell contact to Zeb1 expression, proliferation and dedifferentiation in retinal pigment epithelial cells. Invest Ophthalmol Vis Sci. 2010;51(7):3372-3378.

51. Al-Hussaini H, Schneiders M, Lundh P, Jeffery G. Drusen are associated with local and distant disruptions to human retinal pigment epithelium cells. Exp Eye Res. 2009;88(3):610-612.

52. Rhee J, Buchan T, Zukerberg L, Lilien J, Balsamo J. Cables links Robo-bound Abl kinase to N-cadherin-bound beta-catenin to mediate Slit-induced modulation of adhesion and transcription. Nat Cell Biol. 2007;9(8):883-892.

53. Warburg O. On respiratory impairment in cancer cells. Science. 1956;124(3215):269-270.

54. Thiery JP, Acloque H, Huang RY, Nieto MA. Epithelial-mesenchymal transitions in development and disease. Cell. 2009;139(5):871-890.

55. Yang Y, et al. A selective small molecule inhibitor of c-Met, PHA-665752, reverses lung premalignancy induced by mutant K-ras. Mol Cancer Ther. 2008;7(4):952-960

56. Kaplan O, Firon M, Vivi A, Navon G, Tsarfaty I. HGF/SF activates glycolysis and oxidative phosphorylation in DA3 murine mammary cancer cells. Neoplasia. 2000;2(4):365-377.

57. Cross DA, Alessi DR, Cohen P, Andjelkovich M, Hemmings BA. Inhibition of glycogen synthase kinase- 3 by insulin mediated by protein kinase B. Nature. 1995;378(6559):785-789.

58. Nelson WJ, Nusse R. Convergence of Wnt, betacatenin, and cadherin pathways. Science. 2004; 303(5663):1483-1487.

59. Datta SR, et al. Akt phosphorylation of BAD couples survival signals to the cell-intrinsic death machinery. Cell. 1997;91(2):231-241.

60. Nave BT, Ouwens M, Withers DJ, Alessi DR, Shepherd PR. Mammalian target of rapamycin is a direct target for protein kinase B: identification of a convergence point for opposing effects of insulin and amino-acid deficiency on protein translation. Biochem J. 1999;344(pt 2):427-431.

61. Wullschleger S, Loewith R, Hall MN. TOR signaling in growth and metabolism. Cell. 2006; 124(3):471-484.

62. DeBerardinis RJ, Lum JJ, Hatzivassiliou G, Thompson CB. The biology of cancer: metabolic reprogramming fuels cell growth and proliferation. Cell Metab. 2008;7(1):11-20.

63. Porstmann T, et al. SREBP activity is regulated by mTORC1 and contributes to Akt-dependent cell growth. Cell Metab. 2008;8(3):224-236.

64 . Wang L, et al. Abundant lipid and protein components of drusen. PLoS One. 2010;5(4):e10329.

65. Van Aken EH, De Wever O, Van Hoorde L, Bruyneel E, De Laey JJ, Mareel MM. Invasion of retinal pigment epithelial cells: $\mathrm{N}$-cadherin, hepatocyte growth factor, and focal adhesion kinase. Invest Ophthalmol Vis Sci. 2003;44(2):463-472.

66. Enzmann V, et al. Behavioral and anatomical abnormalities in a sodium iodate-induced model of retinal pigment epithelium degeneration. Exp Eye Res. 2006;82(3):441-448.

67. Li Y, et al. Retinal pigment epithelium damage enhances expression of chemoattractants and migration of bone marrow-derived stem cells. Invest Ophthalmol Vis Sci. 2006;47(4):1646-1652.

68. Jones RG, Thompson CB. Tumor suppressors and cell metabolism: a recipe for cancer growth. Genes Dev. 2009;23(5):537-548.

69. Martin KA, et al. The mTOR/p70 S6K1 pathway regulates vascular smooth muscle cell differentiation. Am J Physiol Cell Physiol. 2004;286(3):C507-C517.

70. Aitken KJ, et al. Mammalian target of rapamycin (mTOR) induces proliferation and de-differentiation responses to three coordinate pathophysiologic stimuli (mechanical strain, hypoxia, and extracellular matrix remodeling) in rat bladder smooth muscle. Am J Pathol. 2009;176(1):304-319.

71. Punzo C, Kornacker K, Cepko CL. Stimulation of the insulin/mTOR pathway delays cone death in a mouse model of retinitis pigmentosa. Nat Neurosci. 2009;12(1):44-52.

72. Zhang H, et al. Trafficking of membrane-associated proteins to cone photoreceptor outer segments requires the chromophore 11-cis-retinal. J Neurosci. 2008;28(15):4008-4014.

73. Wenzel A, Reme CE, Williams TP, Hafezi F, Grimm C. The Rpe65 Leu450Met variation increases retinal resistance against light-induced degeneration by slowing rhodopsin regeneration. J Neurosci. 2001; 21(1):53-58.

74. Bruban J, et al. Amyloid-beta(1-42) alters structure and function of retinal pigmented epithelial cells. Aging Cell. 2009;8(2):162-177.

75. Gnana-Prakasam JP, et al. Absence of iron-regulatory protein $\mathrm{Hfe}$ results in hyperproliferation of retinal pigment epithelium: role of cystine/glutamate exchanger. Biochem J. 2009;424(2):243-252.

76. Hadziahmetovic M, et al. Ceruloplasmin/hephaestin knockout mice model morphologic and molecular features of AMD. Invest Ophthalmol Vis Sci. 2008;49(6):2728-2736.

77. Justilien $\mathrm{V}$, et al. SOD2 knockdown mouse model of early AMD. Invest Ophthalmol Vis Sci. 2007; 48(10):4407-4420.

78. Arnold JJ, Sarks JP, Killingsworth MC, Kettle EK, Sarks SH. Adult vitelliform macular degeneration: a clinicopathological study. Eye (Lond). 2003;17(6):717-726

79. Liu B, Faia L, Hu M, Nussenblatt RB. Pro-angiogenic effect of IFNgamma is dependent on the PI3K/ $\mathrm{mTOR} /$ translational pathway in human retinal pigmented epithelial cells. Mol Vis. 2010;16:184-193.

80. De S, Rabin DM, Salero E, Lederman PL, Temple S, Stern JH. Human retinal pigment epithelium cell changes and expression of alphaB-crystallin: a biomarker for retinal pigment epithelium cell change in age-related macular degeneration. Arch Ophthalmol. 2007;125(5):641-645.

81. Carracedo A, et al. Inhibition of mTORC1 leads to MAPK pathway activation through a PI3K-dependent feedback loop in human cancer. J Clin Invest. 2008;118(9):3065-3074.

82. Kinkade CW, et al. Targeting AKT/mTOR and ERK MAPK signaling inhibits hormone-refractory prostate cancer in a preclinical mouse model. J Clin Invest. 2008;118(9):3051-3064.

83. Xu AW, et al. Effects of hypothalamic neurodegeneration on energy balance. PLoS Biol. 2005;3(12):e415.

84. Prasad D, et al. TAM receptor function in the retinal pigment epithelium. Mol Cell Neurosci. 2006; 33(1):96-108.

85. Zhao C, et al. Autosomal-dominant retinitis pigmentosa caused by a mutation in SNRNP200, a gene required for unwinding of U4/U6 snRNAs. Am J Hum Genet. 2009;85(5):617-627.

86. Livak KJ, Schmittgen TD. Analysis of relative gene expression data using real-time quantitative PCR and the 2(-Delta Delta C(T)) Method. Methods. 2001; 25(4):402-408.

87. Schaeren-Wiemers N, Gerfin-Moser A. A single protocol to detect transcripts of various types and expression levels in neural tissue and cultured cells: in situ hybridization using digoxigenin-labelled cRNA probes. Histochemistry. 1993;100(6):431-440.

88. Strick DJ, Feng W, Vollrath D. Mertk drives myosin II redistribution during retinal pigment epithelial phagocytosis. Invest Ophthalmol Vis Sci. 2009; 50(5):2427-2435

89. Paskowitz DM, et al. Rapid and stable knockdown of an endogenous gene in retinal pigment epithelium. Hum Gene Ther. 2007;18(10):871-880.

90. Zeng LH, Xu L, Gutmann DH, Wong M. Rapamycin prevents epilepsy in a mouse model of tuberous sclerosis complex. Ann Neurol. 2008;63(4):444-453. 